\title{
Physical characteristics of bright Class I methanol masers
}

\author{
S. Leurini ${ }^{1}$, K. M. Menten ${ }^{1}$, and C. M. Walmsley ${ }^{2,3}$ \\ 1 Max-Planck-Institut für Radioastronomie, Auf Dem Hügel 69, 53121 Bonn, Germany \\ e-mail: sleurini@mpifr-bonn.mpg.de \\ 2 INAF-Osservatorio Astrofisico di Arcetri, Largo E. Fermi 5, 50125 Firenze, Italy \\ 3 Dublin Institute of Advanced Studies, 31 Fitzwilliam Place, 2 Dublin, Ireland
}

Received 15 December 2015 / Accepted 11 May 2016

\begin{abstract}
Context. Class I methanol masers are thought to be tracers of interstellar shock waves. However, they have received relatively little attention mostly as a consequence of their low luminosities compared to other maser transitions. This situation has changed recently and Class I methanol masers are now routinely used as signposts of outflow activity especially in high extinction regions. The recent detection of polarisation in Class I lines now makes it possible to obtain direct observational information about magnetic fields in interstellar shocks.

Aims. We make use of newly calculated collisional rate coefficients for methanol to investigate the excitation of Class I methanol masers and to reconcile the observed Class I methanol maser properties with model results.

Methods. We performed large velocity gradient calculations with a plane-parallel slab geometry appropriate for shocks to compute the pump and loss rates which regulate the interactions of the different maser systems with the maser reservoir. We study the dependence of the pump rate coefficient, the maser loss rate, and the inversion efficiency of the pumping scheme of several Class I masers on the physics of the emitting gas.

Results. We predict inversion in all transitions where maser emission is observed. Bright Class I methanol masers are mainly hightemperature $(>100 \mathrm{~K})$ high-density $\left(n\left(\mathrm{H}_{2}\right) \sim 10^{7}-10^{8} \mathrm{~cm}^{-3}\right)$ structures with methanol maser emission measures, $\xi$, corresponding to high methanol abundances close to the limits set by collisional quenching. Our model predictions reproduce reasonably well most of the observed properties of Class I methanol masers. Class I masers in the $25 \mathrm{GHz}$ series are the most sensitive to the density of the medium and mase at higher densities than other lines. Moreover, even at high density and high methanol abundances, their luminosity is predicted to be lower than that of the $44 \mathrm{GHz}$ and $36 \mathrm{GHz}$ masers. Our model predictions also reflect the observational result that the $44 \mathrm{GHz}$ line is almost always stronger than the $36 \mathrm{GHz}$ maser. By comparison between observed isotropic photon luminosities and our model predictions, we infer maser beam solid angles of roughly $10^{-3}$ steradian.

Conclusions. We find that the Class I masers can reasonably be separated into three families: the $(J+1)_{-1}-J_{0}-E$ type series, the $(J+1)_{0}-J_{1}-A$ type, and the $J_{2}-J_{1}-E$ lines at $25 \mathrm{GHz}$. The $25 \mathrm{GHz}$ lines behave in a different fashion from the other masers as they are only inverted at high densities above $10^{6} \mathrm{~cm}^{-3}$ in contrast to other Class I masers. Therefore, the detection of maser activity in all three families is a clear indication of high densities.
\end{abstract}

Key words. ISM: molecules - masers - stars: formation

\section{Introduction}

As first realised by Batrla et al. (1987), methanol masers come in two varieties, termed Class I and II by Menten (1991a,b). Class II methanol masers are closely associated with high-mass young stellar objects (YSOs) and arise from the hot molecular layers heated by the embedded YSOs before and after the formation of an ultracompact HII region. When they occur after the formation of an ultracompact HII region, they frequently coexist with hydroxyl (OH) masers (Menten et al. 1992) and are, like the OH masers (Cesaroni \& Walmsley 1991), most likely pumped by far-infrared radiation in dense $\left(n \sim 10^{7} \mathrm{~cm}^{-3}\right)$ warm $(T \sim 150 \mathrm{~K})$ gas (Sobolev \& Deguchi 1994a,b; Sobolev et al. 1997). In contrast, Class I masers are collisionally pumped and their occurrence can be explained from the basic properties of the $\mathrm{CH}_{3} \mathrm{OH}$ molecule (Lees 1973).

The presence of a Class II methanol maser associated with a star forming core provides unambiguous and sufficient proof of the presence of a high-mass protostellar or young stellar object (Urquhart et al. 2015), and more than a thousand of these sources have been found in Galaxy-wide surveys (Green et al. 2009).
In contrast, until recently relatively little attention was paid to Class I methanol masers. However, recent observational results have changed this situation. Cyganowski et al. (2009) show that these masers are associated with shocked material in protostellar outflows and thus trace very early stages of massive star formation. It is also worth noting that Class I masers have also been detected from a few outflows associated with low-mass protostars (Kalenskii et al. 2010a). Moreover, Sjouwerman et al. (2010b) and Pihlström et al. (2014) report the detection of Class I methanol masers towards supernova remnants (see Frail 2011, for an overview).

Additionally, Sarma \& Momjian $(2009,2011)$ report the discovery of circular polarisation in the 36 and $44 \mathrm{GHz}$ Class I maser lines, which they interpret as a result of the Zeeman effect. These results suggest that Class I masers could be exploited to measure magnetic fields in interstellar shocks and could deliver inputs for magneto-hydrodynamic modelling of shock models. From the point of view of modelling, recent progress in the calculation of collisional rate coefficients has resulted in numbers that now allow meaningful modelling of methanol excitation 
over a wide range of interstellar conditions (Rabli \& Flower 2010).

The aim of our study is to investigate the properties of bright Class I methanol masers focussing mainly on masers with brightness temperatures higher than the critical value corresponding to the saturated intensity $J_{\mathrm{s}}$ beyond which maser amplification becomes linear rather than exponential (see Elitzur 1992; Hollenbach et al. 2013). These masers are usually associated with regions of massive star formation, while lower luminosity Class I masers are reported in supernovae remnants (e.g., Pihlström et al. 2014). We assume the masers to be pumped by collisions with the para form of $\mathrm{H}_{2}$ and compute the pump and decay rates, as well as the pump efficiency for the most commonly observed Class I methanol maser transitions. An implicit assumption is that the escape probabilities of the relevant transitions populating the upper and lower maser levels are highly anisotropic allowing coherence along the line of sight, while a large velocity gradient in the plane of the sky allows relatively high escape probabilities in other directions. This type of velocity field is expected to be produced by a shock propagating perpendicular to the line of sight; moreover, a shock of this kind is expected to produce a maser volume of high aspect ratio oriented along the line of sight. The shock is also expected to cause a rise in the methanol abundance as is suggested by some of the observational data discussed in Sect. 2 of this article.

In this context, we have concentrated on geometry independent maser characteristics and their dependence on physical properties such as density and temperature. We have thus computed the maser output per cubic centimetre and have estimated the densities and methanol abundances at which maser action is quenched. We do not, on the other hand, attempt to compute maser beaming angles and brightness temperatures, although we do focus on size scales (roughly 10-300 AU) corresponding to the observed maser diameters. Our hope is that our results in combination with future interferometric work will allow an increased understanding of the structure of interstellar shocks and the dissipation of mechanical energy in regions of star formation. In a follow-up study, we plan to implement geometry effects in our model to compute brightness temperatures and beaming angles and properly model observations of Class I masers in massive star forming regions.

In Sect. 2 we give a review of the observational properties of Class I methanol masers, followed in Sect. 3 by a description of previous studies on the pumping mechanism for Class I masers. In Sect. 4 we present our model for the pumping of Class I methanol masers, and compute the photon production rates of high-gain masers as a function of the physical parameters of the gas. In Sect. 5, we compare our model results with the observed properties of Class I masers. In Sect. 6 we conclude with possible observational and theoretical perspectives to improve our knowledge of Class I masers.

\section{Phenomenology of Class I methanol masers}

After the first example of a Class I methanol maser was discovered, the $25 \mathrm{GHz} J_{k=2}-J_{k=1} E$ transitions in Orion-KL (Barrett et al. 1971), it took 15 years before these lines were found in other regions (Menten et al. 1986), and before the $44 \mathrm{GHz} 7_{0} \rightarrow 6_{1}-A^{+}$and the $36 \mathrm{GHz} 4_{-1} \rightarrow 3_{0}-E$ methanol masers (Morimoto et al. 1985) were discovered. This paucity of detections of the $25 \mathrm{GHz}$ lines was partially a result of the masers' relatively modest luminosities and because they are frequently found offset from traditional tracers of star formation such as strong infrared sources and ultracompact HII regions
(Menten et al. 1986). As discussed above, an association between Class I methanol masers and protostellar outflows was established later on.

Table 1 reports all known Class I methanol maser transitions to date. For reference, examples of spectra of Class I masers are given in Fig. 1 towards DR21-W and NGC 2264 where the $4_{-1} \rightarrow 3_{0} E$ line also shows broader thermal emission. The $44 \mathrm{GHz} 7_{0} \rightarrow 6_{1}-A^{+}$and $95 \mathrm{GHz} 8_{0} \rightarrow 77_{1}-A^{+}$lines are generally the most widespread and the strongest Class I transitions observed (e.g., Haschick et al. 1990; Val'tts et al. 2000), and therefore they have traditionally been the target of searches for Class I methanol masers in astronomical sources (e.g., Bachiller et al. 1990; Slysh et al. 1994; Kurtz et al. 2004; Ellingsen 2005; Cyganowski et al. 2009; Chen et al. 2011). The next most widespread Class I masers are the $36 \mathrm{GHz} 4_{-1} \rightarrow$ $3_{0}-E$ and $84 \mathrm{GHz} 5_{-1} \rightarrow 4_{0}-E$ transitions (e.g., Haschick \& Baan 1989; Kalenskii et al. 2001; Voronkov et al. 2014). Recent observations at high angular resolution (Voronkov et al. 2007; Britton \& Voronkov 2012) show that even the $25 \mathrm{GHz}$ masers are more common than previously thought. The new capabilities of the upgraded VLA and ATCA interferometers allow the simultaneous observations of several transitions in the $J_{2} \rightarrow J_{1} E$ series thus allowing better investigation of these masers. The masers at $9.9 \mathrm{GHz}, 23.4 \mathrm{GHz}$, and $104 \mathrm{GHz}$ are rare and they have been detected in only a handful of objects (see Voronkov et al. 2012). Interestingly, many of the remaining Class I masers fall within ALMA bands.

\subsection{Occurrence of Class I methanol masers}

In contrast to Class II masers, which typically coincide with hot molecular cores, ultracompact HII regions, $\mathrm{OH}$ masers, and near-IR sources (e.g., Menten et al. 1988a,b), Class I methanol masers are frequently found in the general vicinity of intermediate- and high-mass star formation, but often significantly offset (up to a parsec) from prominent centres of activity, such as infrared sources or compact radio sources (Menten et al. 1986). Based on large samples of sources, Kurtz et al. (2004) and Voronkov et al. (2014) concluded that the vast majority of Class I masers at 44 and $36 \mathrm{GHz}$ are within $1 \mathrm{pc}$ of a Class II methanol maser at $6.7 \mathrm{GHz}$, taken as indicative of the position of the YSO. We note, incidentally, that the only two cases reported by Kurtz et al. (2004) where Class I masers coincide with Class II features are observed with coarse angular resolution $(\sim 0.1-0.2 \mathrm{pc})$.

Plambeck \& Menten (1990) suggested that Class I methanol masers trace the swept-up interaction region of outflows with ambient material. This scenario is supported by an increasing number of observations that reveal a coincidence between Class I masers and other molecular shock tracers (e.g., Johnston et al. 1992; Kurtz et al. 2004; Voronkov et al. 2006, 2010a). Cyganowski et al. (2009) surveyed a sample of massive young stellar objects with extended $4.5 \mu \mathrm{m}$ emission attributed to shocked $\mathrm{H}_{2}$ in outflows and found a detection rate of $44 \mathrm{GHz}$ Class I methanol masers of $\sim 89 \%$. In addition, Class I masers also seem to be associated with molecular outflows from lowmass young stellar objects (Kalenskii et al. 2010a,b). It is important to note that Class I masers may be a unique tool for investigating outflow activity where other standard shock tracers are too weak to be detected. Figure 2, for example, shows a zoom in DR21 $(\mathrm{OH})$ where several Class I maser lines clearly trace bow shocks in a bipolar outflow (Araya et al. 2009) not detected in Spitzer $\mathrm{H}_{2}$ observations likely owing to extinction (Davis et al. 2007). 
Table 1. Known interstellar Class I methanol maser transitions.

\begin{tabular}{|c|c|c|c|}
\hline Transition & Frequency $(\mathrm{MHz})$ & Lab. Ref. & Astronomical reference \\
\hline $9_{-1} \rightarrow 8_{-2}-E$ & 9936 & L2 & A28 \\
\hline $10_{1} \rightarrow 9_{2}^{-} A^{-}$ & 23445 & L5 & A48 \\
\hline $3_{2} \rightarrow 3_{1}-E$ & 24929 & L3 & $\mathrm{A} 12, \mathrm{~A} 20$ \\
\hline $4_{2} \rightarrow 4_{1}-E$ & 24933 & L1 & $\mathrm{A} 1, \mathrm{~A} 6, \mathrm{~A} 8, \mathrm{~A} 12, \mathrm{~A} 20$ \\
\hline $2_{2} \rightarrow 2_{1}-E$ & 24934 & L1 & A7, A12, A17, A20 \\
\hline $5_{2} \rightarrow 5_{1}-E$ & 24959 & L4 & $\mathrm{A} 1, \mathrm{~A} 6, \mathrm{~A} 12, \mathrm{~A} 17, \mathrm{~A} 20, A 24$ \\
\hline $6_{2} \rightarrow 6_{1}-E$ & 25018 & L4 & $\begin{array}{l}\mathrm{A} 1, \mathrm{~A} 2, \mathrm{~A} 3, \mathrm{~A} 17, \mathrm{~A} 20, \mathbf{A} 4 *, \mathrm{~A} 5, \mathrm{~A} 6, \mathrm{~A} 7, A 8, \mathbf{A 9}, \mathrm{A} 12, \\
\mathbf{A 1 3}, \mathrm{A} 17, \mathrm{~A} 20, A 24, \mathbf{A 2 6} *, A 36, \mathbf{A 3 8 *}\end{array}$ \\
\hline $7_{2} \rightarrow 7_{1}-E$ & 25125 & L4 & $\mathrm{A} 1, \mathrm{~A} 2, \mathrm{~A} 3, \mathrm{~A} 17, \mathrm{~A} 20$ \\
\hline $8_{2} \rightarrow 8_{1}-E$ & 25294 & L4 & $\mathrm{A} 1, \mathrm{~A} 6, \mathrm{~A} 17$ \\
\hline $9_{2} \rightarrow 9_{1}-E$ & 25541 & L4 & $\mathrm{A} 12, \mathrm{~A} 32$ \\
\hline $10_{2} \rightarrow 10_{1}-E$ & 25878 & L4 & A39 \\
\hline $12_{2} \rightarrow 12_{1}-E$ & 26847 & L5 & A32 \\
\hline $13_{2} \rightarrow 13_{1}-E$ & 27473 & L5 & A32 \\
\hline $14_{2} \rightarrow 14_{1}-E$ & 28169 & L5 & A32 \\
\hline $15_{2} \rightarrow 15_{1}-E$ & 28906 & L5 & A32 \\
\hline $16_{2} \rightarrow 16_{1}-E$ & 29637 & L5 & A32 \\
\hline $17_{2} \rightarrow 17_{1}-E$ & 30308 & L5 & A32 \\
\hline $4_{-1} \rightarrow 3_{0}-E$ & 36169 & L5 & A4, A9, A10, A13, A19, A25, A26, A27, A33, A51 \\
\hline $7_{0} \rightarrow 6_{1}-A^{+}$ & 44069 & L5 & $\mathbf{A 4}, \mathbf{A 9}, \mathrm{A} 10, \mathrm{~A} 21, \mathrm{~A} 22, \mathbf{A 2 6}, \mathrm{A} 30, \mathrm{~A} 34, \mathrm{~A} 37, A 44, A 46, \mathrm{~A} 47, A 51$ \\
\hline $5_{-1} \rightarrow 4_{0}-E$ & 84521 & L3 & $A 14, \mathbf{A 1 3}, \mathbf{A 2 6}, \mathrm{A} 42$ \\
\hline $8_{0} \rightarrow 7_{1}-A^{+}$ & 95169 & L3 & $\mathrm{A} 11, \mathrm{~A} 18, A 23, \mathbf{A 2 6}, \mathrm{A} 29, \mathrm{~A} 31, \mathrm{~A} 49, \mathrm{~A} 50$ \\
\hline $11_{-1} \rightarrow 10_{-2}-E$ & 104300 & L3 & 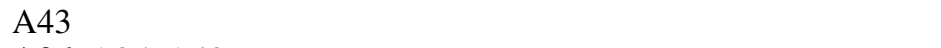 \\
\hline $6_{-1} \rightarrow 5_{0}-E$ & 132891 & L5 & A26, A35, A40 \\
\hline $9_{0} \rightarrow 8_{1}-A^{+}$ & 146618 & L5 & A26 \\
\hline $4_{2} \rightarrow 3_{1}-E$ & 218440 & L6 & A52 \\
\hline $8_{-1} \rightarrow 7_{0} E$ & 229758 & L6 & A43 \\
\hline $9_{-1} \rightarrow 8_{0}-E$ & 278305 & L6 & $A 53$ \\
\hline
\end{tabular}

References. References are in chronological order. Laboratory references for higher accuracy frequencies are: L1: Gaines et al. (1974); L2: Breckenridge \& Kukolich (1995); L3: Müller et al. (2004); L4: Tsunekawa et al. (1995); L5: Mehrotra et al. (1985); L6: Sastry et al. (1984). Astronomical references presenting interferometric observations are given in italics, theoretical papers in boldface. Theoretical references discussing various of the $J_{2} \rightarrow J_{1}-E$ transitions are only listed for the $6_{2} \rightarrow 6_{1}-E$ transition and are marked by an asterisk. Astronomical references are: A1: Barrett et al. (1971); A2: Chui et al. (1974); A3: Hills et al. (1975); A4*: Pelling (1975); A5: Barrett et al. (1976) (VLBI); A6: Barrett et al. (1975); A7: Buxton et al. (1977); A8: Matsakis et al. (1980); A9: Sobolev \& Strelnitskii (1983); A10: Morimoto et al. (1985); A11: Nakano \& Yoshida (1986); A12: Menten et al. (1986); A13: Zeng et al. (1987); A14: Batrla \& Menten (1988); A17: Menten et al. (1988d,c); A18: Plambeck \& Wright (1988); A19: Haschick \& Baan (1989); A20: Menten \& Batrla (1989); A21: Haschick et al. (1990); A22: Bachiller et al. (1990); A23: Plambeck \& Menten (1990); A24: Johnston et al. (1992); A25: Kalenskii et al. (1992); A26*: Cragg et al. (1992); A27: Haschick \& Baan (1989); A28: Slysh et al. (1993); A29: Kalenskii et al. (1994); A30: Slysh et al. (1994); A31: Val'tts et al. (1995); A32: Wilson et al. (1996); A33: Liechti \& Wilson (1996); A34: Mehringer \& Menten (1997); A35: Slysh et al. (1997); A36: Johnston et al. (1997); A37: Kogan \& Slysh (1998); A38*: Matsakis et al. (1980); A39: Sobolev et al. (1998); A40: Slysh et al. (1999); A41: Val'tts et al. (2000); A42: Kalenskii et al. (2001); A43: Slysh et al. (2002); A44: Kurtz et al. (2004); A45: Voronkov et al. (2005); A46: Cyganowski et al. (2009); A47: Fontani et al. (2010); A48: Voronkov et al. (2011); A49: Chen et al. (2011); A50: Gan et al. (2013); A51: Voronkov et al. (2014); A52: Hunter et al. (2014); A53: Yanagida et al. (2014).

A location in the swept-up interaction region of outflows with ambient material may be a natural explanation for some of the observed properties of Class I masers. In C-shocks we expect that the high relative velocities of charged grains and neutral gas cause methanol ice to be sputtered from the grain surface and released into the gas leading to the high methanol abundance and column density required for observable maser emission. Usually, no high-velocity Class I maser emission is observed and the observed features are clustered within a few $\mathrm{km} \mathrm{s}^{-1}$ around the ambient velocity. These features can be explained by the fact that paths moving perpendicular to the line of sight have the longest velocity-coherent gain length and have, naturally, velocities close to $v_{\mathrm{LSR}}$. However, it should be noted that Voronkov et al. (2014) recently reported a small number of high-velocity Class I maser components, which are largely blueshifted and detected more easily at $36 \mathrm{GHz}$.
Class I masers, however, do not exclusively trace molecular outflows. They have also been detected in other astrophysical environments in the presence of shocked gas, for example at the interaction of supernova remnants with the molecular cloud (e.g., Szczepanski et al. 1989; Haschick et al. 1990; Pihlström et al. 2011), in cloud-cloud collisions (e.g., Sobolev 1992), and in layers where expanding HII regions interact with the ambient molecular environment (Voronkov et al. 2010b). Widespread Class I methanol masers have been observed towards the Galactic Centre (Yusef-Zadeh et al. 2013). In this case, the authors have proposed that induced photodesorption by cosmic rays is responsible for the emission instead of large-scale shocks. Recently, Ellingsen et al. (2014) has reported the first tentative detection of Class I maser emission at $36 \mathrm{GHz}$ in the nearby galaxy NGC 253. This detection would be intriguing since so far only the two Class II methanol maser lines that are by far the most 


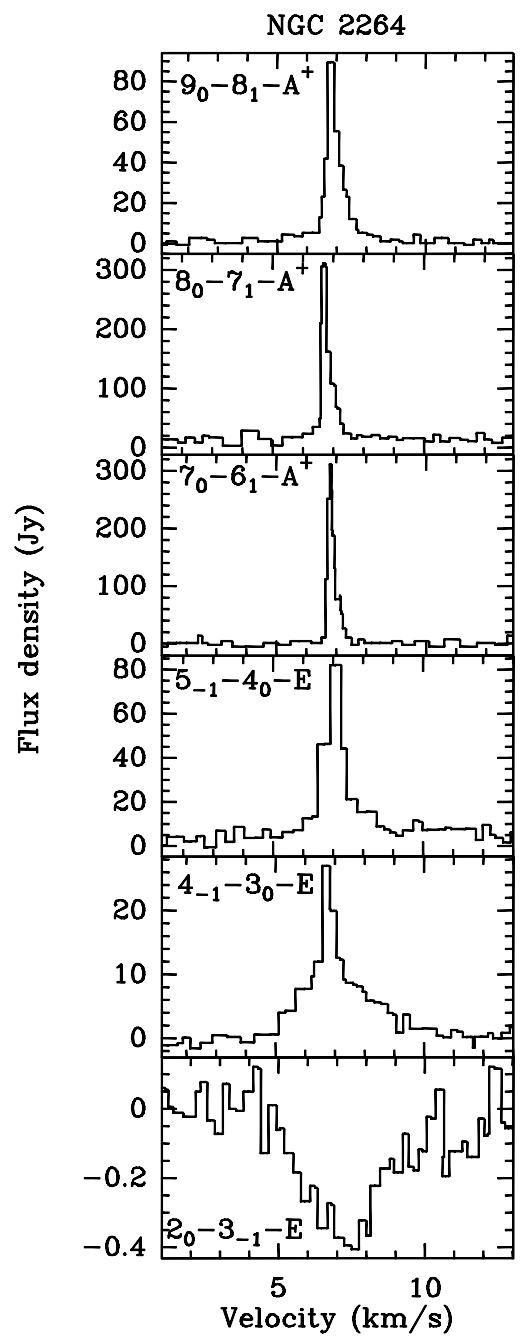

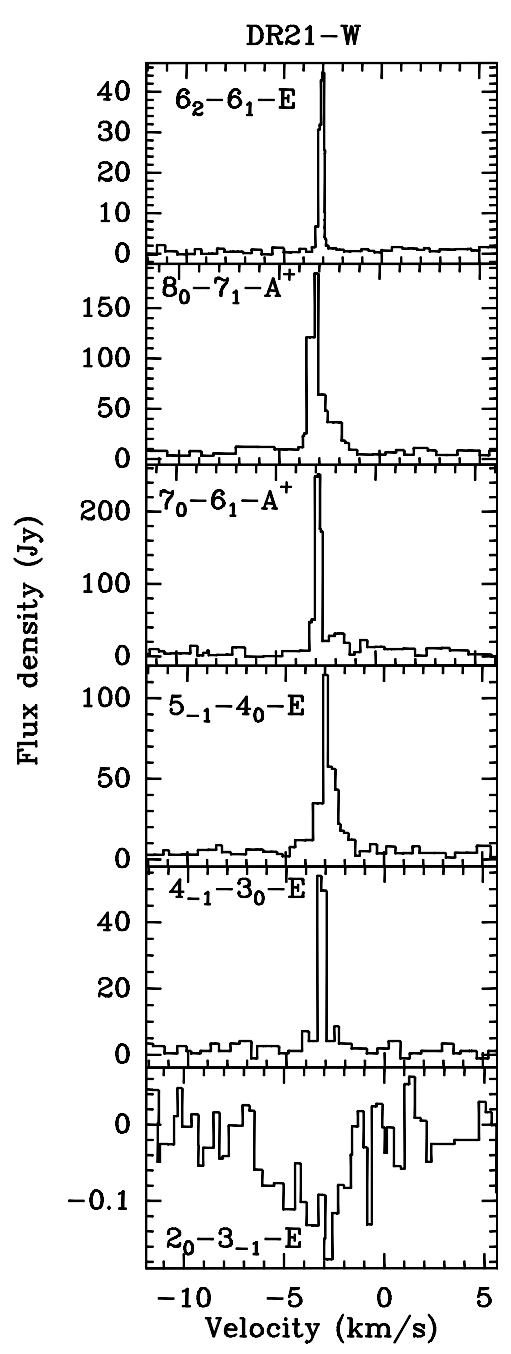

Fig. 1. For both NGC 2264 (left) and DR21-W (right), lowest spectrum: absorption in the $\mathrm{CH}_{3} \mathrm{OH} 2_{0} \rightarrow 3_{-1}-E$ line; other spectra: various maser transitions taken with differing velocity resolutions and a number of telescopes. Adopted from Menten (1991a). All spectra where taken between 1988 and 1990. The spectra of the $4_{-1} \rightarrow 3_{0}-E$ and $7_{0} \rightarrow 6_{1}-A^{+}$lines are from Haschick et al. (1990) and those of the $2_{0} \rightarrow 3_{-1}-E$ lines were taken with the Green Bank 140-foot telescope. The remaining spectra were taken with the IRAM $30 \mathrm{~m}$ telescope. prominent in our Galaxy (the $6.7 \mathrm{GHz}$ and $12.2 \mathrm{GHz}$ lines, e.g., Menten 1991b) have been detected in a few nearby galaxies (Green et al. 2008; Sjouwerman et al. 2010a; Ellingsen et al. 2010).

\subsection{Class I methanol maser spot sizes}

Estimates of spot sizes for Class I masers have in general been based upon VLA measurements and VLBI non-detections (e.g., Barrett et al. 1976; Lonsdale et al. 1998). For example, Johnston et al. (1997) used VLA observations of the $6_{2}-6_{1}-E$ line at $25.018 \mathrm{GHz}$ to conclude that spot sizes in Orion-KL were in the range 22-56 AU. Slysh \& Kalenskii (2009) have also reported that the $44 \mathrm{GHz}$ maser has average spot sizes of $50 \mathrm{AU}$ in OMC-2 and in NGC 2264, and have concluded that Class I maser spots are much larger than Class II maser spots, which have typical sizes of a few AU (Moscadelli et al. 1999; Sanna et al. 2010), implying brightness temperatures of the order of $10^{12} \mathrm{~K}$.

Recently, Matsumoto et al. (2014) detected the $44 \mathrm{GHz}$ maser in the massive star forming region IRAS 18151-1208 for the first time with a VLBI experiment. They found that most of the maser emission is not due to a compact point source, but originates from a spatially extended structure which accounts for about $73 \%$ of the total flux of the maser. The strongest maser feature has a linear size of $\sim 15 \mathrm{AU} \times 9 \mathrm{AU}$ and a brightness temperature of $\sim 10^{10} \mathrm{~K}$, but this should be regarded as a lower limit to the real spot size given the severe filtering of extended emission that affects the data. We note that several masers may have a compact high brightness temperature core surrounded by a larger envelope (hundreds of AU) of lower brightness.

The sizes and brightness temperatures measured for the other features are listed in Table 2 together with typical isotropic luminosities for different Class I masers from the literature. The scatter in the most commonly observed lines at $36 \mathrm{GHz}$ and $44 \mathrm{GHz}$ can be larger than reported as large samples were observed in these transitions. For the $25 \mathrm{GHz}$ series, values reported for the brightness temperature and the spot size are for the $6_{2}-6_{1}$ line only and are measured in Orion-KL. For all lines the reported brightness temperatures and spot sizes are based on interferometric observations and are in most of the cases based on only one source. In the following discussion, we will assume spot sizes of $50 \mathrm{AU}$ and $100 \mathrm{AU}$.

\subsection{Are Class I masers saturated?}

Observed brightness temperatures are upwards of $10^{6} \mathrm{~K}$ when observed with high linear resolution and linear sizes in the range 10-100 AU (see Table 2), whereas the expected limiting brightness temperature $T_{\mathrm{s}}$ for an unsaturated maser (see Sect. 5.2) is typically $10^{5}\left(4 \pi / \Omega_{\mathrm{b}}\right) \mathrm{K}$, where $\Omega_{\mathrm{b}}$ is the maser beam solid angle. Moreover, saturated masers are expected to show little or no evidence of variation. Indeed, saturated masers undergo linear amplification, whereas unsaturated masers should amplify exponentially. This suggests qualitatively that unsaturated masers 


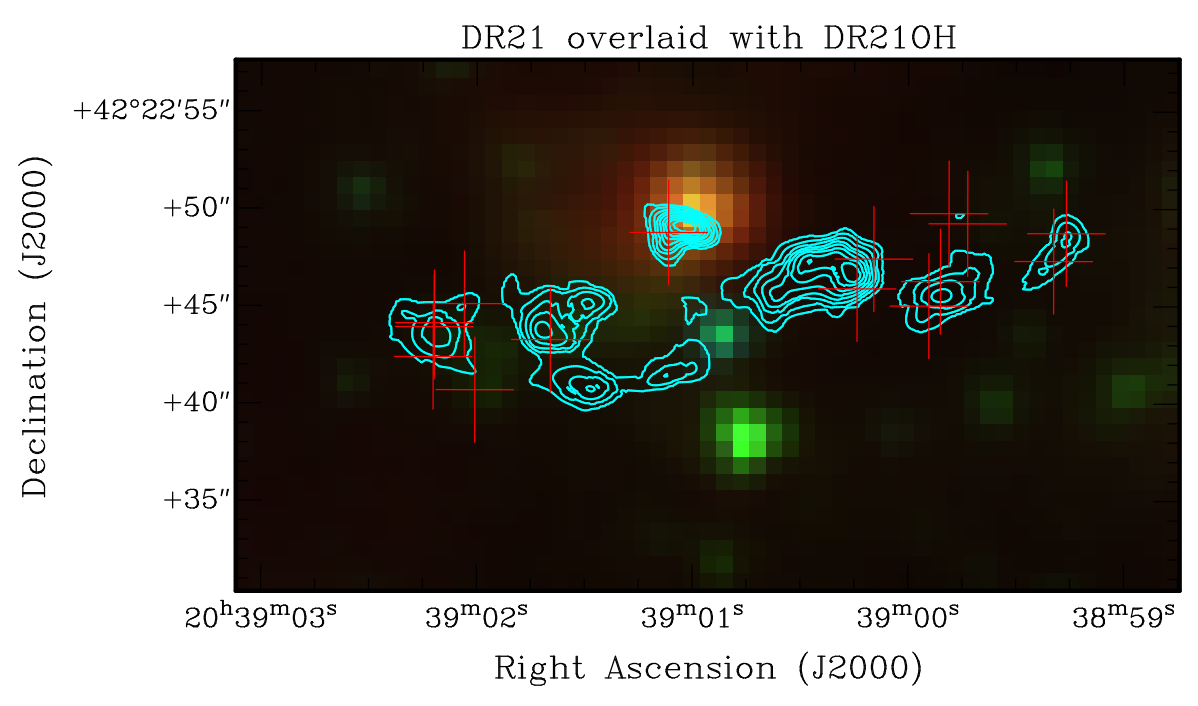

Fig. 2. Spitzer IRAC image of the DR21(OH) region $(3.6 \mu \mathrm{m}$ blue, $4.5 \mu \mathrm{m}$ green, $8.0 \mu \mathrm{m}$ red). The images were retrieved from the Spitzer archive. The red crosses mark the positions of $36 \mathrm{GHz}$ masers from Fish et al. (2011). Cyan contours represent the integrated intensity of the thermal $4_{2} \rightarrow 3_{1}-E$ methanol line (Zapata et al. 2012) which traces the E-W outflow seen in the Class I masers.

Table 2. Summary of typical Class I maser luminosities, $L_{\mathrm{iso}}$, brightness temperatures, $T_{\mathrm{b}}$, and spot sizes.

\begin{tabular}{lllrl}
\hline \hline Line & $\begin{array}{l}L_{\text {iso }} \\
\text { photons s }\end{array}$ & $\begin{array}{l}T_{\mathrm{b}} \\
\mathrm{K}\end{array}$ & $\begin{array}{c}\text { Spot sizes } \\
\text { AU }\end{array}$ & Source \\
\hline $9.9 \mathrm{GHz}$ & $10^{43 a}$ & $10^{4 a}$ & & \\
$25 \mathrm{GHz}$ series & $10^{41}-10^{43} a, b, c, d$ & $2 \times 10^{6}-3 \times 10^{7 b}$ & $22-56^{b}$ & Orion-KL $^{b}$ \\
$36 \mathrm{GHz}$ & $10^{43}-10^{44} e$ & & & \\
$44 \mathrm{GHz}$ & $10^{42}-10^{45} f, g$ & $4 \times 10^{7}-10^{10 g, h}$ & $6-50^{g, h}$ & IRAS 18151-1208 $^{g}$ \\
$84 \mathrm{GHz}$ & $3 \times 10^{42}-2 \times 10^{43 a} a$ & $6 \times 10^{3}-5 \times 10^{5} a$ & & \\
$95 \mathrm{GHz}$ & $5 \times 10^{42}-10^{45 a, i, j, k}$ & $2 \times 10^{4}-10^{5} a$ & & \\
$104 \mathrm{GHz}$ & $2 \times 10^{43 a}$ & $2 \times 10^{4 a}$ & & \\
\hline
\end{tabular}

Notes. Maser luminosities are taken from interferometric studies. Spot sizes are from the VLA observations of Johnston et al. (1997) at $25 \mathrm{GHz}$, and from the VLBI detection of Matsumoto et al. (2014) and are to be considered as upper and lower limits, respectively. The measurements reported by Voronkov et al. (2006) are based on linear resolutions of 4000-6000 AU.

References. ${ }^{(a)}$ Voronkov et al. (2006). ${ }^{(b)}$ Johnston et al. (1997) for the $6_{2}-6_{1}$ line. ${ }^{\left({ }^{c}\right)}$ Menten et al. (1986). ${ }^{(d)}$ Johnston et al. (1992). ${ }^{(e)}$ Fish et al. (2011). ${ }^{(f)}$ Kurtz et al. (2004). ${ }^{(g)}$ Matsumoto et al. (2014). ${ }^{(h)}$ Slysh \& Kalenskii (2009). ${ }^{(i)}$ Moscadelli et al. (2007). ${ }^{(j)}$ Plambeck \& Menten (1990). ${ }^{(k)}$ Chen et al. (2011).

should vary on much shorter time scales than their saturated counterparts. A reasonable guess for the variation time scale of a saturated maser would be $l_{\text {spot }} / v_{\text {sh }}$, where $l_{\text {spot }}$ is the maser spot size and $v_{\text {sh }}$ is the shock velocity. Taking $l_{\text {spot }}=100 \mathrm{AU}$ and a shock velocity of $30 \mathrm{~km} \mathrm{~s}^{-1}$, we find a time scale of $\sim 15 \mathrm{yrs}$. This is consistent with most observations (Menten et al. 1988c; Kurtz et al. 2004) which show that Class I masers have little time variation. Dedicated observations of Class I masers to detect variability over time scales of several years are lacking, however, and variability of Class I masers is basically still an uncharted territory.

We also note that theory suggests that with increasing amplification, unsaturated masers are expected first to narrow and then to re-broaden until the line width approaches the intrinsic value determined by local turbulent and thermal broadening. However, observationally, the line profiles of different Class I masers are found to be very similar (e.g., Batrla \& Menten 1988; Plambeck \& Menten 1990; Voronkov et al. 2014). In particular for the DR21(OH) region, interferometric observations show that all $84 \mathrm{GHz}$ components have counterparts in the $95 \mathrm{GHz}$ line at identical velocities (e.g., Plambeck \& Menten 1990) and that several transitions are spatially coincident (from the $36 \mathrm{GHz}$ line to the $229 \mathrm{GHz}$ maser, Fish et al. 2011, their Fig. 1 and references therein). It is worth noting, however, that real observed maser emission is likely a mixture of both saturated and unsaturated contributions. Taken all together, the observational properties seem to suggest that bright Class I masers are at least partially saturated.

\section{Previous excitation studies on Class I masers}

Previous statistical equilibrium studies on $\mathrm{CH}_{3} \mathrm{OH}$ Class I masers made use of collisional rates based on experimental results by Lees \& Oka (1969) and Lees \& Haque (1974). These rates show a propensity for $\Delta k=0$ collisions and a dependence upon $\Delta J$ as $1 / \Delta J$. The propensity for $\Delta k=0$ over $\Delta k=1$ collisions causes molecules to preferentially de-excite down a $k$ stack (see Fig. 3), leading to a situation where the lower levels in the $k=-1$ stack are overpopulated relative to those in the $k=0$ stack (for $\mathrm{CH}_{3} \mathrm{OH}-E$ ), resulting in maser action in the $J_{-1} \rightarrow(J-1)_{0}-E$ series and enhanced absorption in the $2_{0} \rightarrow 3_{-1} E$ line, the second most prominent Class II maser line. Similar mechanisms lead to inversion in the $9_{-1} \rightarrow 8_{-2}$ and $11_{-1} \rightarrow 10_{-2}-E$ lines. Analogously, the $K=0$ ladder of $\mathrm{CH}_{3} \mathrm{OH}-\mathrm{A}$ is overpopulated, leading to the $J_{0} \rightarrow(J-1)_{1}-A^{+}$ masers and to the anti-inversion of the $5_{1} \rightarrow 6_{0} A^{+}$Class II transition, the strongest Class II maser line. Indeed, observations showed that the $2_{0} \rightarrow 3_{-1} E$ and $5_{1} \rightarrow 6_{0} A^{+}$Class II lines are detected in absorption toward a number of Class I sources (e.g., Batrla et al. 1987; Menten 1991b) and also in dark clouds (Walmsley et al. 1988). 


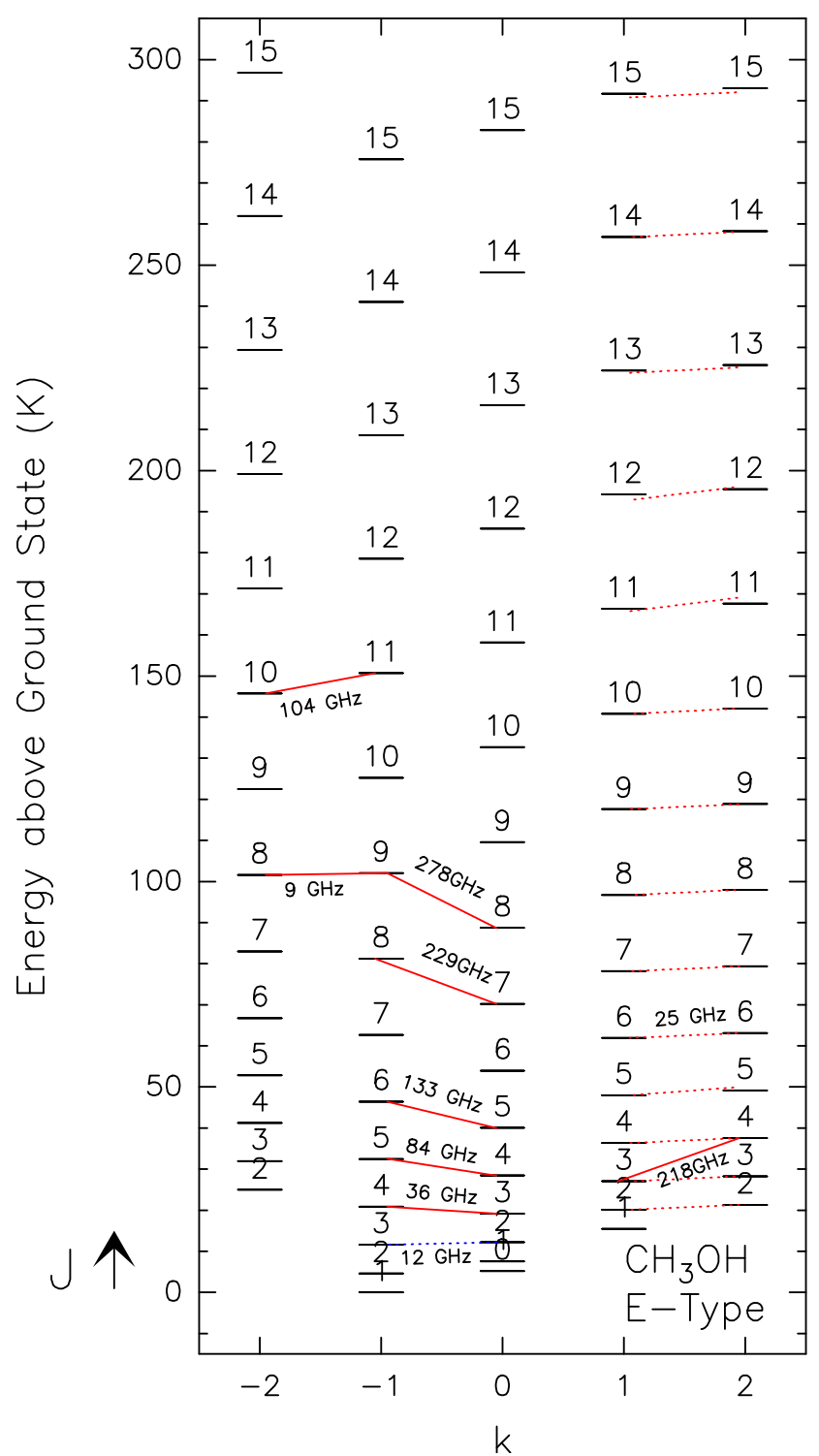

(a)

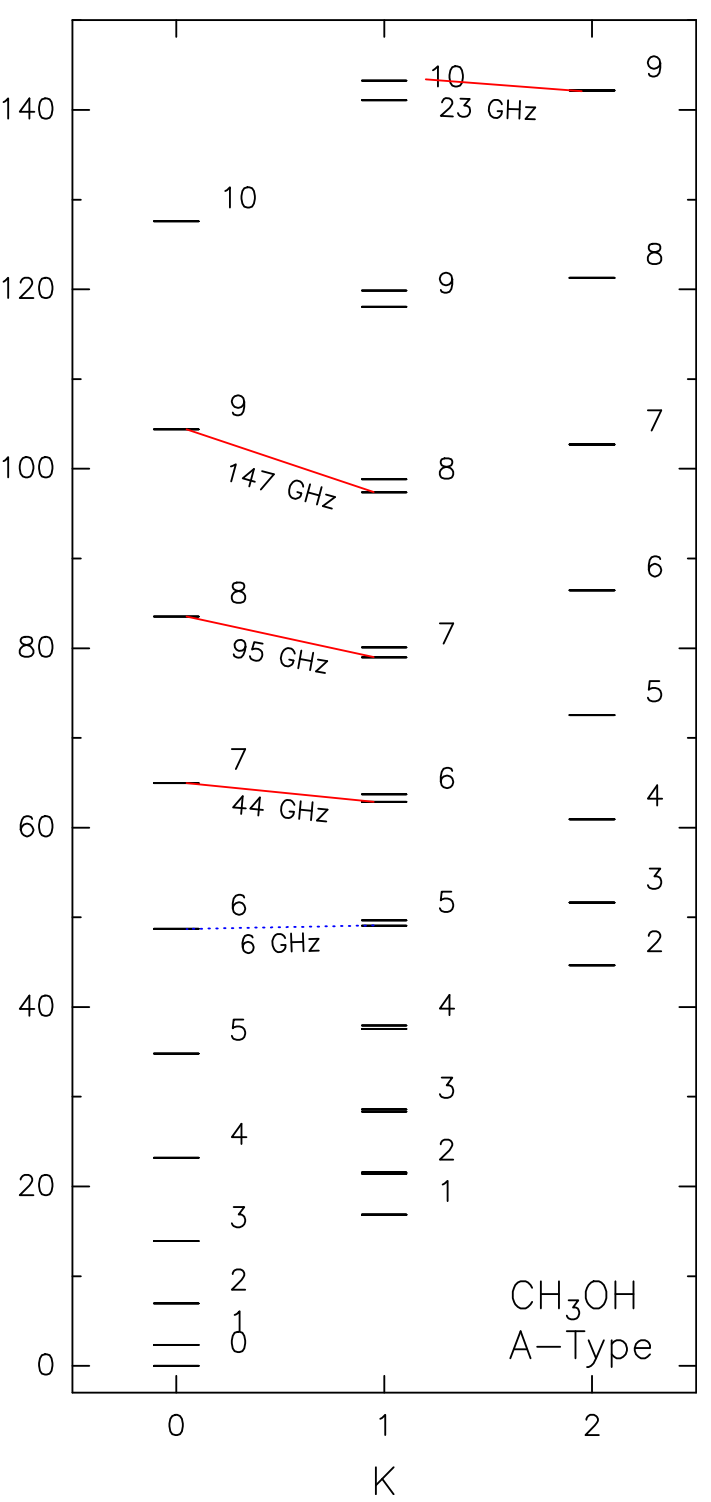

(b)

Fig. 3. Partial rotational level diagram of $E$-type (left) and $A$-type methanol (right). Known Class I maser transitions are connected by red lines with frequencies indicated; blue dotted lines connect anti-inverted transitions. We note that all of the maser lines originating in the $J_{2} \rightarrow J_{1} E$ series near $25 \mathrm{GHz}$ are indicated by dotted red lines.

However, while the previously used collisional rates can reproduce the anti-inversion at $12.18 \mathrm{GHz}$ and $6.7 \mathrm{GHz}$ and several Class I masers $\left(4_{-1} \rightarrow 3_{0} E\right.$ at $36 \mathrm{GHz}, 5_{-1} \rightarrow 4_{0} E$ at $84 \mathrm{GHz}$, $7_{0} \rightarrow 6_{1} A^{+}$at $44 \mathrm{GHz}, 8_{0} \rightarrow 7_{1} A^{+}$at $95 \mathrm{GHz}$, and $9_{0} \rightarrow 8_{1} A^{+}$ $146 \mathrm{GHz}$; see Fig. 3), they do not account for the Class I maser series $J_{2} \rightarrow J_{1} E$ at $25 \mathrm{GHz}$. For this reason, Johnston et al. (1992) suggested an ad hoc additional preference for the $\Delta k=3$ collisions and the assumption that $\Delta k=2$ collisions are not allowed to be able to collisionally pump the $J_{2} \rightarrow J_{1} E$ Class I masers. Alternatively, Cragg et al. (1992) modelled the pumping of interstellar methanol masers using the hard sphere model of Goldreich \& Kwan (1974), which represents the extreme case of completely unselective collisions. In this case, all Class I masers, including the $J_{2} \rightarrow J_{1} E$ series, appear for densities in the range $10^{2}-10^{5} \mathrm{~cm}^{-3}$.

The general conclusion of previous models of Class I masers is that these lines are collisionally excited and that they mase in different density ranges. These general results have recently been confirmed by McEwen et al. (2014) and Nesterenok (2016).

\section{Statistical equilibrium results}

We performed large velocity gradient (LVG) calculations using a modified version of the code presented by Leurini et al. (2004) with a plane-parallel slab geometry appropriate for shocks. Following a standard formalism (Elitzur 1992), the maser levels are described by pump $\left(P_{\mathrm{m}}\right)$ and loss $\left(\Gamma_{\mathrm{m}}\right)$ rates that regulate the interactions of the different maser systems with all other levels (which we refer to as the maser reservoir)

$$
\begin{aligned}
& P_{\mathrm{m}}=\sum_{i \neq m} n_{i} \times\left(A_{i m} \times \beta_{i m}+C_{i m} \times n\right) \\
& \Gamma_{\mathrm{m}}=\sum_{i \neq m}\left(A_{\mathrm{mi}} \times \beta_{\mathrm{mi}}+C_{\mathrm{mi}} \times n\right),
\end{aligned}
$$


where $m=1,2$ are the two levels of the maser system, $n_{i}$ the $\mathrm{CH}_{3} \mathrm{OH}$ density in level $i, n$ the molecular hydrogen density, $A_{i m}$ the Einstein coefficients for spontaneous emission (with $A_{\text {im }}=0$ for $i<m), C_{i m}$ the temperature dependent collisional rates, $\beta_{i m}$ the escape probability $\left(\beta_{i m}=\frac{\left(1-\exp \left(-3 \tau_{i m}\right)\right)}{3 \tau_{i m}}\right)$, for a slab geometry, and $\tau_{i m}$ the optical depth (perpendicular to the line of sight) given by Eq. (2) of Leurini et al. (2004). The pump and loss rates are obtained by solving the level populations for the full system (maser and reservoir) in the absence of maser radiation. We also note that we neglect in these calculations the continuum dust opacity when computing $\beta_{i m}$ and thus assume that the opacity at wavelengths of transitions feeding maser levels is small.

We updated the code with the more recent collisional rates for the torsional ground states of $A$ - and $E$-type methanol from Rabli \& Flower (2010). The rates were computed for temperatures in the range from 10 to $200 \mathrm{~K}$ including rotational states up to $J=15$ for collisions with para- $\mathrm{H}_{2}$. We assume in this study that collisions with ortho- $\mathrm{H}_{2}$ can be neglected or, equivalently, that the abundance ratio of ortho- to para- $\mathrm{H}_{2}$ is low. This needs to be investigated in future work, but we note that if the ratio of ortho- to para- $\mathrm{H}_{2}$ is low in the pre-shock gas, this may also hold in the post-shock layer because the time taken to traverse the shock may be too short to thermalise the ratio. Indeed, Wilgenbus et al. (2000) found that significant conversion from para- to ortho- $\mathrm{H}_{2}$ starts at $700 \mathrm{~K}$ in C-type shocks (and at $T>1000 \mathrm{~K}$ in $J$-type).

We ran models for densities between $10^{4}$ and $10^{9} \mathrm{~cm}^{-3}$ and temperatures, $T$, of $20-400 \mathrm{~K}$. Since collisional de-excitation rates from Rabli \& Flower (2010) are computed for temperatures up to $200 \mathrm{~K}$, we use the rates computed at $200 \mathrm{~K}$ for higher temperatures. We used specific column densities $(N / \Delta v)$ of $\mathrm{CH}_{3} \mathrm{OH}-$ $A$ and $-E$ between $10^{14}$ and $10^{18} \mathrm{~cm}^{-2} \mathrm{~km}^{-1} \mathrm{~s}$. In the absence of any external radiation field except the cosmic background, all known Class I maser transitions should be reproduced and the Class II $2_{0} \rightarrow 3_{-1} E$ and $5_{1} \rightarrow 6_{0} A^{+}$masers should be antiinverted. All Class I transitions reported in Table 2 show inversion in the analysed range of physical conditions. In addition, the $2_{0} \rightarrow 3_{-1} E$ transition and the $5_{1} \rightarrow 6_{0} A^{+}$line present antiinversion as expected. In particular, the range of densities for which the lines are anti-inverted depends on the temperature and column density. For the same value of $T$, the $2_{0} \rightarrow 3_{-1} E$ line is anti-inverted at densities lower than the $5_{1} \rightarrow 6_{0} A^{+}$transition. The absorption temperature increases with methanol column density and kinetic temperature.

In the following discussion, we present the pumping mechanisms that lead to maser action in the Class I lines and the dependency of the masers on the physics of the gas. We should note that although the general behaviour is similar to the results recently published by McEwen et al. (2014), our calculations produce inversion in the $36 \mathrm{GHz}$ line at lower densities $\left(10^{4}-10^{5} \mathrm{~cm}^{-3}\right)$ than found by McEwen et al. (2014) $\left(10^{6} \mathrm{~cm}^{-3}\right)$. The main difference between our models and those by McEwen et al. (2014) is that the latter use a $30 \mathrm{~K}$ dust radiation field, while our models include only the cosmic radiation field. This assumption is justified by the fact that Class I masers are usually found far from continuum sources (e.g., Voronkov et al. 2014). To test our results, we benchmarked our code with the RADEX LVG code (van der Tak et al. 2007) with a uniform slab geometry. The results of the two codes are consistent up to a specific column density of $10^{17} \mathrm{~cm}^{-2} \mathrm{~km}^{-1} \mathrm{~s}$.

\subsection{Pump and loss rates}

In this paper, we confine ourselves to calculating the maser efficiency and other characteristics of saturated masers following the formalism used by Hollenbach et al. (2013) for water masers. We define the methanol maser emission measure, $\xi$, which is a measure of the rate at which pump photons are produced. It is defined as the ratio of the product of the molecular hydrogen and methanol number densities over the velocity gradient in the plane of the sky:

$\xi=\frac{X_{6}\left(\mathrm{CH}_{3} \mathrm{OH}\right) \times n_{6}^{2}}{(\mathrm{~d} V / \mathrm{d} r)}$.

Here, $X_{6}\left(\mathrm{CH}_{3} \mathrm{OH}\right)$ is the methanol abundance $\left(n\left(\mathrm{CH}_{3} \mathrm{OH}\right) / n\left(\mathrm{H}_{2}\right)\right)$ in units of $10^{-6}$ (we assume equal abundances for $A$ - and $E$-type methanol), $n_{6}$ the $\mathrm{H}_{2}$ number density in units of $10^{6} \mathrm{~cm}^{-3}$, and $\mathrm{d} V / \mathrm{d} r$ the velocity gradient in units of $\mathrm{km} \mathrm{s}^{-1} \mathrm{pc}^{-1}$ (for rough estimates, we assume a shock velocity of $30 \mathrm{~km} \mathrm{~s}^{-1}$ and a shock width of $10^{16} \mathrm{~cm}$; see Schilke et al. 1997; Gusdorf et al. 2008). We note that $\xi$ can also be expressed in terms of the ratio of methanol column density (in the plane of the sky) to line width, $N\left(\mathrm{CH}_{3} \mathrm{OH}\right) / \mathrm{d} v$ :

$$
\begin{aligned}
& \frac{N\left(\mathrm{CH}_{3} \mathrm{OH}\right)}{\mathrm{d} v}=\frac{3.3 \times 10^{18} \times n\left(\mathrm{CH}_{3} \mathrm{OH}\right)}{\mathrm{d} V / \mathrm{d} r} \\
& \xi=\frac{n\left(\mathrm{H}_{2}\right) \times \frac{N\left(\mathrm{CH}_{3} \mathrm{OH}\right)}{\mathrm{d} v}}{3.3 \times 10^{24}} .
\end{aligned}
$$

To investigate the pumping mechanism of Class I methanol masers, we define effective pump and loss rates of a given maser system as $P=P_{u}-P_{l}$ and $L=\Gamma_{u}-\Gamma_{l}$, and study their behaviour as functions of $\xi$ for different temperatures and densities. As an example, $P$ and $L$ for the $36 \mathrm{GHz}$ lines are represented in Fig. 4, which shows that both the relative population rates of the upper and lower maser levels, as well as their relative decay rates, play a role in causing population inversion. This process does not depend significantly on the temperature, while it does depend on the volume density of the gas, which emphasises the importance of collisions for Class I masers. The other Class I masers behave similarly.

\subsection{Conditions for population inversion}

Figure 5 shows the excitation temperature of the $44 \mathrm{GHz}$ and of the $6_{2}-6_{1}-E 25.018 \mathrm{GHz}$ (representative of the $25 \mathrm{GHz}$ series) masers to illustrate the range of $n$ and $\xi$ where the lines are inverted and where the pump and loss rates are meaningful quantities for a temperature of $200 \mathrm{~K}$. For completeness, similar plots for the $36 \mathrm{GHz}, 84 \mathrm{GHz}$, and $95 \mathrm{GHz}$ lines are shown in Appendix A (Fig. A.1). Table 3 summarises the value of $\xi, \xi_{q}$, at which population inversion is quenched for temperatures of $80 \mathrm{~K}$ and of $200 \mathrm{~K}$ for different Class I maser lines at different densities. From Eq. (5), the specific methanol column density at which each maser line quenches can be derived. At a temperature of $200 \mathrm{~K}$, the $36 \mathrm{GHz}$ line thermalises around a few $10^{17} \mathrm{~cm}^{-2} \mathrm{~km}^{-1} \mathrm{~s}$ independently of density for $n>10^{6} \mathrm{~cm}^{-3}$; a similar behaviour is shown by the $84 \mathrm{GHz}$ lines, which also quenches at $\sim 10^{17} \mathrm{~cm}^{-2} \mathrm{~km}^{-1} \mathrm{~s}$ for all densities. The same is found for the other lines reported in Table 3: they all quench at a constant specific column density of approximately $10^{17} \mathrm{~cm}^{-2} \mathrm{~km}^{-1} \mathrm{~s}$ independently of volume density in the range $10^{5}-10^{7} \mathrm{~cm}^{-3}$, which suggests that there is a critical methanol column density at which quenching occurs. At a lower temperature of $80 \mathrm{~K}$, the specific column density at which the quenching 

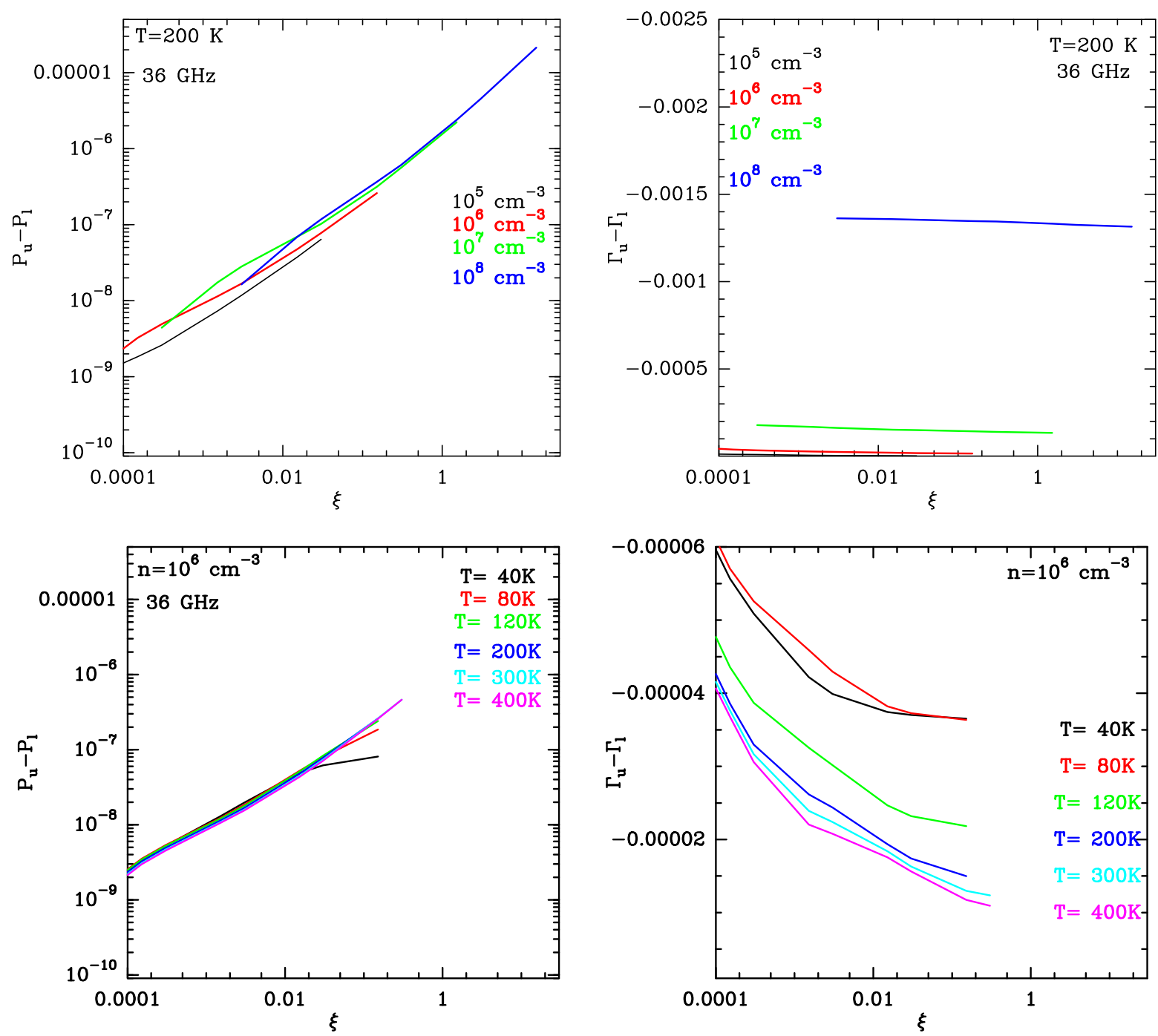

Fig. 4. Effective pump and loss rate for the $36 \mathrm{GHz}$ maser at different densities and for a temperature of $200 \mathrm{~K}$ (upper panels) and at different temperatures for $n=10^{6} \mathrm{~cm}^{-3}$ (lower panels). It is possible to convert $\xi$ to methanol abundance using Eq. (3).

occurs increases to approximately $10^{18} \mathrm{~cm}^{-2} \mathrm{~km}^{-1} \mathrm{~s}$ at a volume density of $10^{8} \mathrm{~cm}^{-3}$ except for the $6_{2}-6_{1}-E 25.018 \mathrm{GHz}$ line. To give a complete view of the range of parameters where the Class I maser lines are inverted, we also plot their excitation temperatures as functions of densities for a temperature of $200 \mathrm{~K}$ and a specific column density of $10^{17} \mathrm{~cm}^{-2} \mathrm{~km}^{-1} \mathrm{~s}$ (Fig. 6). Figure 6 clearly illustrates the effect that varying the volume density has on individual lines: the $25 \mathrm{GHz}$ series quenches at the highest volume density and it is inverted for $n>10^{6} \mathrm{~cm}^{-3}$; the $36 \mathrm{GHz}$, $44 \mathrm{GHz}$, and $95 \mathrm{GHz}$ lines are those inverted over the broadest range of volume densities. Figure 6 also shows that the $84 \mathrm{GHz}$ may be not inverted at low densities (a few $10^{4} \mathrm{~cm}^{-3}$ ). However, this behaviour is found only at high specific column densities $\left(>10^{16} \mathrm{~cm}^{-2} \mathrm{~km}^{-1} \mathrm{~s}\right)$ and in a narrow range of densities.

\subsection{Maser emission}

We follow the formalism developed by Elitzur et al. (1989) and Hollenbach et al. (2013) for $\mathrm{H}_{2} \mathrm{O}$ masers and describe the maser emission through the pump rate coefficient $(q)$, the maser loss rate $(\Gamma)$, and the inversion efficiency of the pumping scheme $(\eta)$ as

$q=\frac{1}{2} \times \frac{P_{u}+P_{l}}{n^{2} \times\left(\mathrm{CH}_{3} \mathrm{OH}\right)}$

$\Gamma=\frac{g_{u} \Gamma_{u}+g_{l} \Gamma_{l}}{g_{u}+g_{l}}$

$\eta=\frac{P_{u} \Gamma_{l}-P_{l} \Gamma_{u}}{P_{u} \Gamma_{l}+P_{l} \Gamma_{u}}$

Here $g_{u}$ and $g_{l}$ are the statistical weights for the upper and lower level of a given maser system. We note that our definition of $\eta$ is different from that of Hollenbach et al. (2013) because we do not assume that the upper and lower levels of a given maser system have the same decay rates.

Figures 7-9 show our results for the $44 \mathrm{GHz}$, the $36 \mathrm{GHz}$, and the $25.018 \mathrm{GHz}$ masers for $\eta, q$, and $\Gamma / n_{6}$ (the loss rate divided by density in units of $10^{6} \mathrm{~cm}^{-3}$ ) as functions of $\xi$ for a range of densities and temperatures relevant for Class I masers. The parameters $\Gamma$ and $q$ are the sum of a collisional part and a radiative part: at low $\xi$, collisions are negligible and $\Gamma$ and $q$ are dominated by their radiative part, which decreases with increasing values of $\xi$. Their collisional part is proportional to the $\mathrm{H}_{2}$ density and has a weak dependence on temperature. Hence, $\Gamma / n_{6}$ is fairly flat as a function of $\xi$ when collisions dominate, 

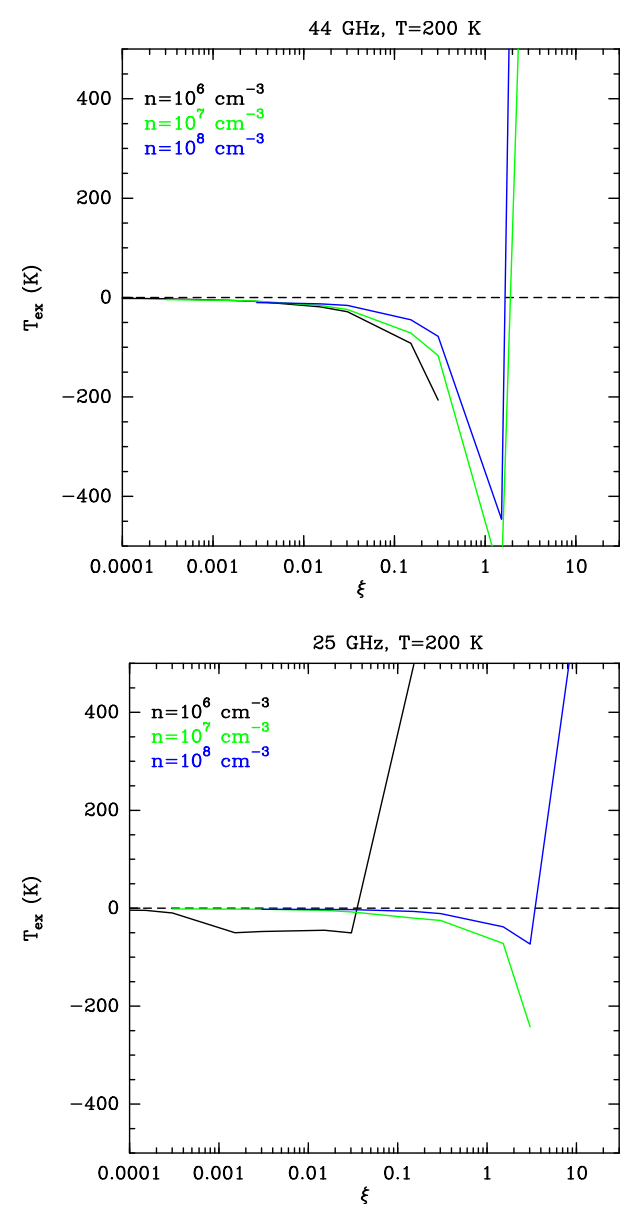

Fig. 5. Excitation temperature, $T_{\mathrm{ex}}$, of the $44 \mathrm{GHz}$ (upper panel) and of the $6_{2}-6_{1}-E 25.018 \mathrm{GHz}$ line (lower panel) at different densities and for a temperature of $200 \mathrm{~K}$. Curves are plotted until $T_{\mathrm{ex}}$ becomes positive. It is possible to convert $\xi$ to methanol abundance using Eq. (3). We note that with increasing $\xi$, the excitation temperature typically transits from -infinity to +infinity before thermalising. The discrete nature of our grid in $\xi$ occasionally obscures this fact.

while $q$ decreases slowly with $\xi$ in this regime. Results for the other Class I maser lines are shown in Appendix A, Figs. A.2 and A.3. For the $44 \mathrm{GHz}$ and the $95 \mathrm{GHz}$ lines, $\Gamma, q$, and $\eta$ have a very weak dependence on density and on temperature (above $80 \mathrm{~K}$ ) as function of $\xi$ (Figs. 7 and A.3). On the other hand, the $36 \mathrm{GHz}, 84 \mathrm{GHz}$, and the $25 \mathrm{GHz}$ lines show a stronger dependency on density; the $25 \mathrm{GHz}$ line is the most sensitive to changes in the volume density of the gas (Figs. 8, 9, and A.2). All Class I masers analysed in this study do not show a strong dependence on temperature.

\subsection{Effects of the physical parameters on Class I masers}

The parameters $\eta$ and $q$ discussed in the previous sections can be used to characterise the maser emission (e.g., Hollenbach et al. 2013). The photon production rate $\Phi_{\mathrm{m}}$ at line centre of a saturated maser is directly linked to the observed flux $\left(F_{\mathrm{m}}\right)$ of a given maser through the geometry of the maser and can be defined as

$\Phi_{\mathrm{m}}=\frac{g_{u} g_{l}}{g_{u}+g_{l}} \times 2 n^{2} X\left(\mathrm{CH}_{3} \mathrm{OH}\right) \eta \mathrm{q}$

Figures 10 and 11 show the dependence of $\Phi_{\mathrm{m}}$ for several Class I methanol lines $(36 \mathrm{GHz}, 44 \mathrm{GHz}, 95 \mathrm{GHz}$, and the $25 \mathrm{GHz}$ series) as functions of $\xi$ for different densities and temperatures for a range of parameters for which the lines are inverted. For the $J_{2}-J_{1}-E$ series we show our model results for the photon production rate for lines between $J=2$ and 10 . The photon production rate of the $84 \mathrm{GHz}$ line is presented in Appendix A (Fig. A.4). For all lines, the photon production rates follow almost a single curve as functions of $\xi$ independently of volume density. All lines are basically unaffected by $T$. For the $25 \mathrm{GHz}$ series, we find that the photon production rate increases with $J$ with a peak between $J=8$ and 10 (see discussion in Sect. 5.3).

In Fig. 12, we compare the photon production rates for the $6_{2} \rightarrow 6_{1}$ line with those of the $36 \mathrm{GHz}$ and $44 \mathrm{GHz}$ masers for densities of $10^{6} \mathrm{~cm}^{-3}, 10^{7} \mathrm{~cm}^{-3}$, and $10^{8} \mathrm{~cm}^{-3}$. Clearly, the $6_{2} \rightarrow 6_{1}$ line (and in general the whole $J_{2} \rightarrow J_{1}$ series at $25 \mathrm{GHz}$, see Fig. 11) behaves in a different fashion from the other maser transitions discussed in this article and is the Class I maser most sensitive to density. This is not surprising. The $36 \mathrm{GHz}, 44 \mathrm{GHz}$, $84 \mathrm{GHz}$, and $95 \mathrm{GHz}$ masers are all directly connected to backbone ladders ( $k=-1$ for $E$-type and $K=0$ for $A$-type) and are inverted mainly because of the predominance of $\Delta k=0$ collisions. This is not the case for the $J_{2} \rightarrow J_{1}$ series whose inversion mechanism is more complex and which depends on $\Delta k \neq 0$ collisions to build up population in the $k=1$ and $2 E$-type ladders. As seen in Fig. 6, the $6_{2}-6_{1}-E$ transition is only inverted at high densities above $10^{6} \mathrm{~cm}^{-3}$ in contrast to the other Class I masers treated here. Moreover, the density dependence of the photon production rate in $6_{2}-6_{1}-E$ is steeper than in backbone-linked lines and the dependence on $\xi$ (emission measure) is flatter. This is clearly shown in Fig. 12 at a density of $10^{7} \mathrm{~cm}^{-3}$ where the 36 and $44 \mathrm{GHz}$ masers have photon production rates that are very similar and behave roughly as $\xi^{0.7}$. The photon production rate for the $6_{2}-6_{1}-E$ line on the other hand is close to the backbone-linked lines at low $\xi$, but has a much flatter $\xi$ dependence (roughly $\xi^{0.4}$ ) and consequently has a much lower photon output at $\xi$ values approaching unity. It is clear from our models that at densities of the order of $10^{6} \mathrm{~cm}^{-3}$ the photon output in the $25 \mathrm{GHz}$ lines is much lower than at $36 \mathrm{GHz}$ or $44 \mathrm{GHz}$. Indeed, observations suggest a much lower photon output at $25 \mathrm{GHz}$ than in backbone lines (e.g., Menten et al. 1986; Haschick et al. 1990; Voronkov et al. 2006), but higher quality interferometric observations are needed to draw conclusions.

It is interesting to note that despite the decrease in $\eta$ and $q$ with $\xi$ (see Fig. 7), the production rate increases with $\xi$ until thermalisation is reached, thus suggesting that the high $\xi$ before quenching (corresponding to specific column densities of $10^{16}-10^{17} \mathrm{~cm}^{-2} \mathrm{~km}^{-1} \mathrm{~s}$; see Table 3 and Sect. 4.2) are responsible for the observed bright masers. This implies that high $\xi$ values are needed for high photon production rate and that therefore masers with high brightness temperatures trace high methanol emission measures. These values are reached at high densities, high temperatures, and high methanol column densities (Eq. (3)). In particular, the $36 \mathrm{GHz}$ line can reach higher photon production rates than the $84 \mathrm{GHz}$, the $44 \mathrm{GHz}$, and the $95 \mathrm{GHz}$ masers because it generally quenches at higher values of $\xi$ for $T<200 \mathrm{~K}$. A similar result has been obtained by McEwen et al. (2014) who find (at a temperature of $80 \mathrm{~K}$ and a methanol abundance of $10^{-7}$ ) that optimal masing conditions at $36 \mathrm{GHz}$ are at densities roughly an order of magnitude higher than at $44 \mathrm{GHz}$.

Modelling intensities of saturated maser lines is not straightforward because the outcome depends on the geometry of the maser. In particular, the observed flux of a given maser transition depends on the geometry and on whether the maser is beamed towards us or not. However, under the assumption that different maser lines of frequencies $v_{1}$ and $v_{2}$ are emitted by the same volume of gas and that they are both saturated, the observed ratios 
Table 3. $\xi_{q}$ for various Class I lines at different densities for $T=80 \mathrm{~K}$ and $200 \mathrm{~K}$.

\begin{tabular}{lccccc}
\hline \hline \multicolumn{5}{c}{$T=80 \mathrm{~K}$} \\
\hline Volume density & $36 \mathrm{GHz}$ & $84 \mathrm{GHz}$ & $44 \mathrm{GHz}$ & $95 \mathrm{GHz}$ & $25 \mathrm{GHz}^{a}$ \\
\hline $10^{6} \mathrm{~cm}^{-3}$ & $6 \times 10^{-2}$ & $1 \times 10^{-1}$ & $5 \times 10^{-2}$ & $2 \times 10^{-2}$ & $4 \times 10^{-2}$ \\
$10^{7} \mathrm{~cm}^{-3}$ & 1 & $3 \times 10^{-1}$ & $3 \times 10^{-1}$ & $9 \times 10^{-2}$ & $4 \times 10^{-1}$ \\
$10^{8} \mathrm{~cm}^{-3}$ & $5 \times 10^{-1}$ & $2 \times 10^{-1}$ & $2 \times 10^{-1}$ & $7 \times 10^{-2}$ & 3 \\
\hline \multicolumn{5}{c}{$T=200 \mathrm{~K}$} \\
\hline $10^{6} \mathrm{~cm}^{-3}$ & $2 \times 10^{-1}$ & $3 \times 10^{-2}$ & - & $2 \times 10^{-1}$ & $3 \times 10^{-2}$ \\
$10^{7} \mathrm{~cm}^{-3}$ & $3 \times 10^{-1}$ & $5 \times 10^{-1}$ & 1.9 & 2 & $8 \times 10^{-1}$ \\
$10^{8} \mathrm{~cm}^{-3}$ & 7 & 2 & 1.7 & $5 \times 10^{-1}$ & 3 \\
\hline
\end{tabular}

Notes. ${ }^{(a)}$ We refer here to $6_{2}-6_{1}-E$ transition.
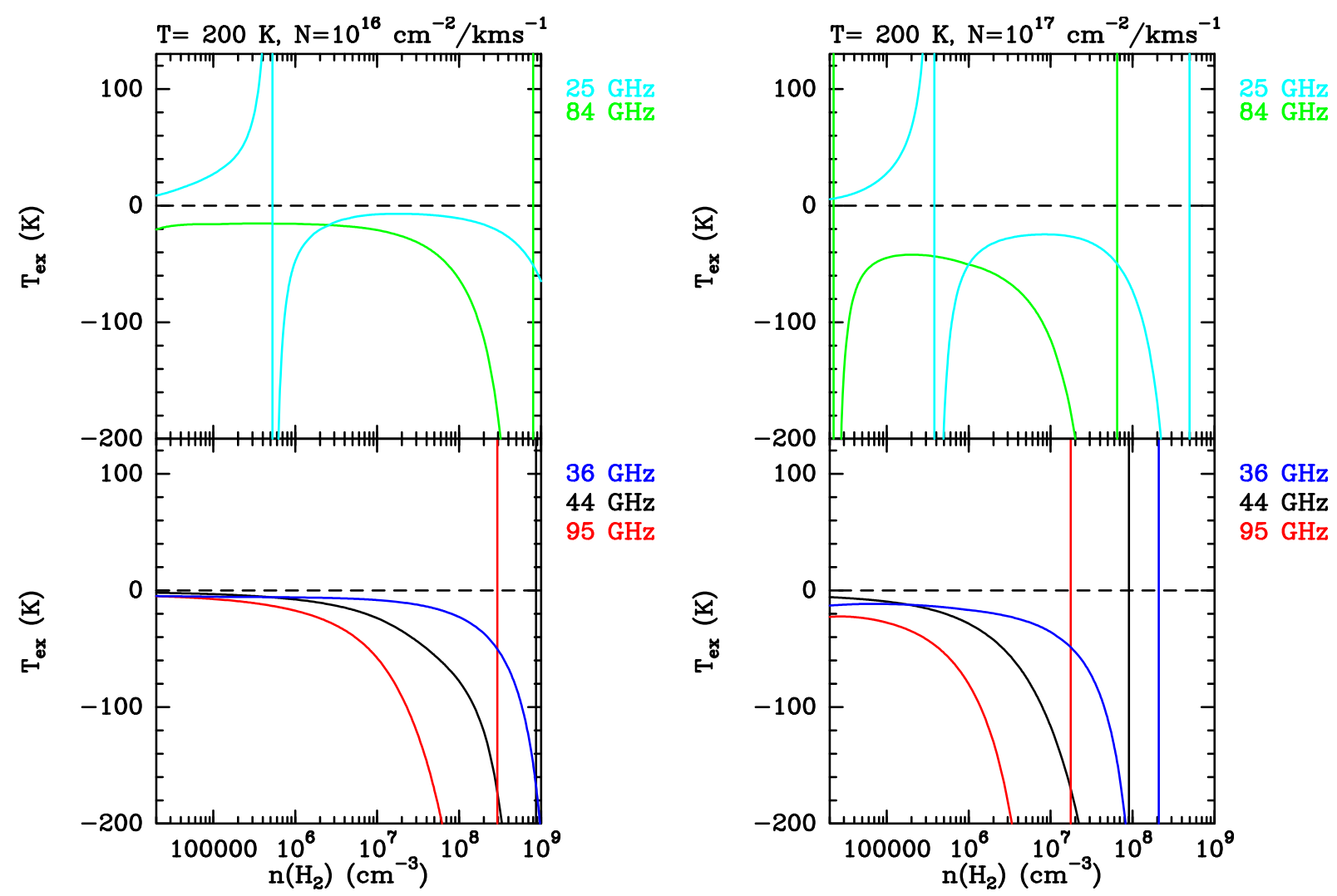

Fig. 6. Excitation temperature of the $44 \mathrm{GHz}, 95 \mathrm{GHz}$, and $36 \mathrm{GHz}$ lines (lower panels), and the $84 \mathrm{GHz}$ and $6_{2}-6_{1}-E$ masers (upper panels) for a temperature of $200 \mathrm{~K}$ and a specific column density of $10^{16} \mathrm{~cm}^{-2} \mathrm{~km}^{-1} \mathrm{~s}($ left $)$ and of $10^{17} \mathrm{~cm}^{-2} \mathrm{~km}^{-1} \mathrm{~s}$ (right) as functions of volume density. The dashed horizontal line separates maser from non-maser behaviour. Vertical lines mark the transitions from maser to non-maser behaviour.

of their fluxes, $F_{1}$ and $F_{2}$, is geometry independent and it can be written as a function of the ratio between their photon production rates:

$\frac{F_{1}}{F_{2}}=\frac{v_{1}}{v_{2}} \frac{\Phi_{1}}{\Phi_{2}}$

Figure 10 shows the $\Phi_{1} / \Phi_{2}$ ratio of the modelled maser photon production rates of the $36 \mathrm{GHz}, 44 \mathrm{GHz}$, and $95 \mathrm{GHz}$ maser lines for different densities and temperature as functions of $\xi$. Figure 12 shows the photon production rate ratios of $6_{2}-6_{1}-E$ transition relative to the $44 \mathrm{GHz}$ and $36 \mathrm{GHz}$ lines. For completeness, the ratio between the $84 \mathrm{GHz}$ maser and the $36 \mathrm{GHz}$ line are shown in Fig. A.4. The ratio $\Phi_{44 \mathrm{GHz}} / \Phi_{95 \mathrm{GHz}}$ is relatively flat and does not vary with density for a fixed temperature of $200 \mathrm{~K}$. On the other hand, it decreases with temperature for a fixed density of $10^{6} \mathrm{~cm}^{-3}$. The ratio $\Phi_{44 \mathrm{GHz}} / \Phi_{36 \mathrm{GHz}}$ has a different behaviour as a function of temperature and density. At a fixed density of $10^{6} \mathrm{~cm}^{-3}$, the ratio increases with $T$, while at $T=$ $200 \mathrm{~K}$ it decreases with volume density. Finally, $\Phi_{36 \mathrm{GHz}} / \Phi_{84 \mathrm{GHz}}$ decreases with density and temperature (Fig. A.4).

\section{Discussion}

\subsection{Maser luminosities}

The luminosity of a given maser transition is defined as

$L_{\mathrm{m}} \propto \Phi_{\mathrm{m}} \times V_{\mathrm{m}}$,

where $V_{\mathrm{m}}$ is the maser volume. As discussed in Sect.2.2, spot sizes for Class I masers are not well constrained. For a 


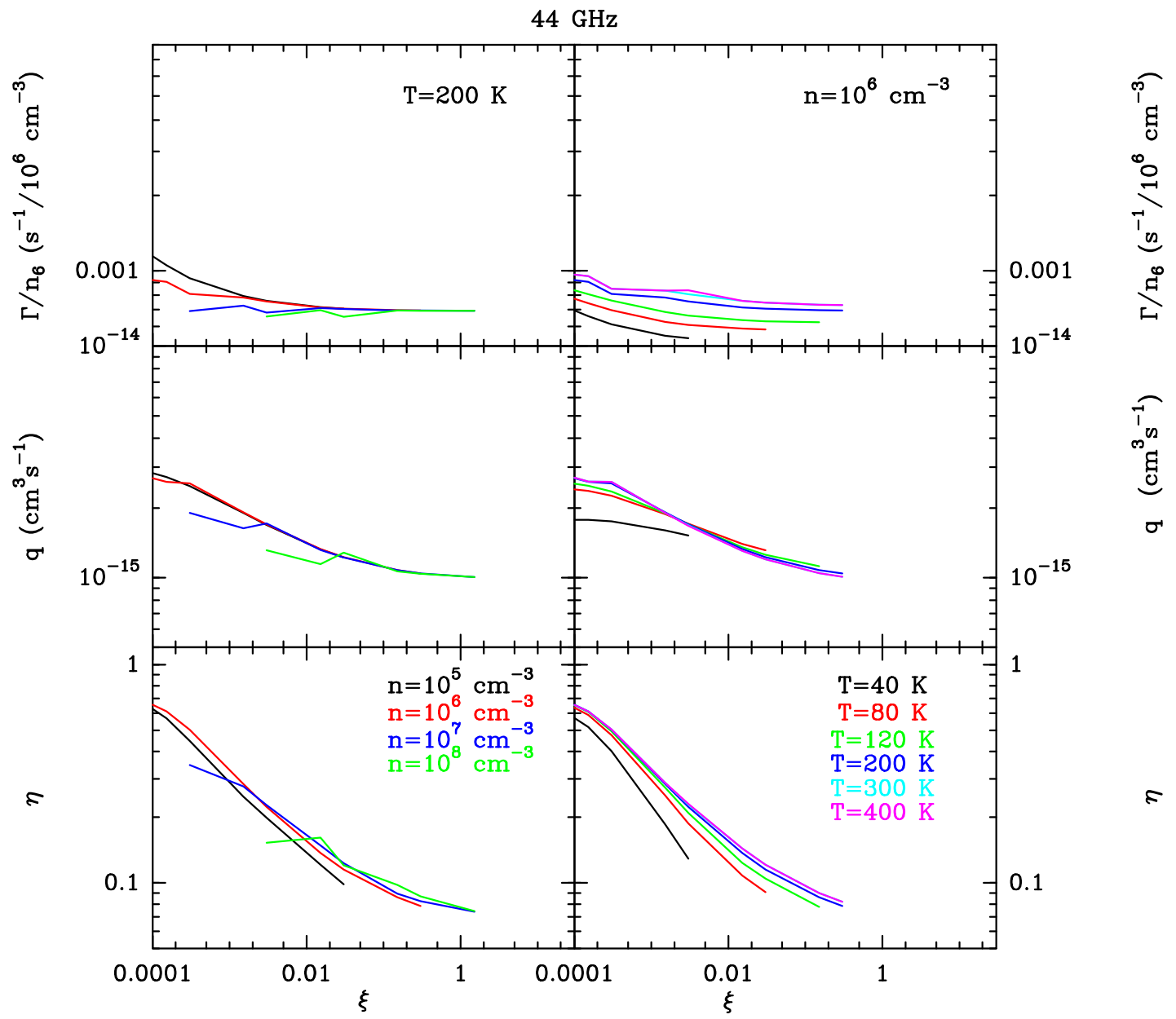

Fig. 7. Modelling results for the $44 \mathrm{GHz}$ maser pumping at $T=200 \mathrm{~K}$ and various densities (left panel) and at $n=10^{6} \mathrm{~cm}^{-3}$ and several temperatures (right panel). Plotted are the maser loss rate $\Gamma$ divided by $n_{6}$, the pump rate coefficient $q$, and the inversion efficiency $\eta$ as functions of $\xi$. It is possible to convert $\xi$ to methanol abundance using Eq. (3).

cylindrical geometry, a spot size of $100 \mathrm{AU}$ and an aspect ratio $\alpha=l_{\text {los }} / R=10$, where $l_{\text {los }}$ is the length oriented along the line of sight and $R$ the radius, we derive maser luminosities for the $44 \mathrm{GHz}, 95 \mathrm{GHz}, 36 \mathrm{GHz}$, and $84 \mathrm{GHz}$ lines, and the $6_{2}-6_{1}-E$ maser (representative of the $25 \mathrm{GHz}$ series) of $10^{41}$ photons s$^{-1}$ for $80 \mathrm{~K}$ and $200 \mathrm{~K}$ for emission measures $\xi$ of 0.1 or 0.2 . The corresponding luminosities for a spot size of $50 \mathrm{AU}$ are approximately $10^{39}$ photons $\mathrm{s}^{-1}$. The maser luminosity is linked to the observed isotropic luminosity, $L_{\text {iso }}$, through the formula

$L_{\mathrm{m}}=L_{\mathrm{iso}} \times \Omega_{\mathrm{b}} /(4 \pi)$,

where $\Omega_{\mathrm{b}}$ is the beaming angle. For a cylindrical maser spot with $l_{\text {los }}=\alpha R$ oriented along the line of sight, $\Omega_{\mathrm{b}}=\pi \times$ $\left(R / l_{\text {los }}\right)^{2}=\pi / \alpha^{2}$ making the assumption that the beam solid angle is purely determined by geometry (not necessarily the case; see Hollenbach et al. 2013 for a discussion). For an aspect ratio $\alpha$ of 10 (corresponding to $\sim 10^{-3}$ steradian), observations of Class I masers $\left(L_{\text {iso }}=10^{41}-10^{45}\right.$ photons s ${ }^{-1}$; see Table 2$)$ imply maser luminosities between between $10^{38}$ and $10^{42}$ photons s ${ }^{-1}$ in agreement with our models.

For a collisionally excited maser, it is thus reasonable to expect that high density and a high methanol abundance is required. This general expectation is confirmed by the results of our computations which show that the maser photon output per unit volume increases with the methanol emission measure $\xi$ defined by Eq. (3). From Eqs. (11) and (12), it can be seen that a small maser volume can be compensated for either by a larger $\Phi_{\mathrm{m}}$ or by a small beaming angle. The former requires higher emission measures (or higher density and methanol abundance), while the latter becomes geometrically unlikely at a certain point (aspect ratios for the masing volume higher than 10 seem improbable). We can get some insights into the implications of this based on our computations of the photon production rates. From Fig. 10, we note that in the most common lines, a photon emissivity per unit volume of $10^{-6}$ photons $\mathrm{s}^{-1} \mathrm{~cm}^{-3}$ at $\xi=0.1$ is achieved, while (see Fig. 12) the $25 \mathrm{GHz}$ emissivity is almost an order of magnitude smaller.

From the methanol emission measure assuming velocity gradients of the order of $5 \times 10^{4} \mathrm{~km} \mathrm{~s}^{-1} \mathrm{pc}^{-1}$ and a standard methanol abundance of $10^{-6}$ (e.g., Menten et al. 1988d; Kama et al. 2010), we find $n\left(\mathrm{H}_{2}\right)=7 \times 10^{7} \mathrm{~cm}^{-3}$ consistent with a high density explanation of the masers.

Such high densities are consistent with the magnetic field measurement of Sarma \& Momjian (2011; see also Momjian \& Sarma 2012). They have detected a Zeeman pattern in the $44 \mathrm{GHz}$ maser towards OMC2 and estimate a line of sight field of $18 \mathrm{mG}$ (with high uncertainty owing to the poorly known Landé factor). If one uses the correlation between density and magnetic field of Crutcher (1999), this corresponds to a $\mathrm{H}_{2}$ density of $10^{7} \mathrm{~cm}^{-3}$, although with a very large uncertainty. We note here that Sarma and Momjian found a value ten times lower based on a different argument and we cannot exclude this. 


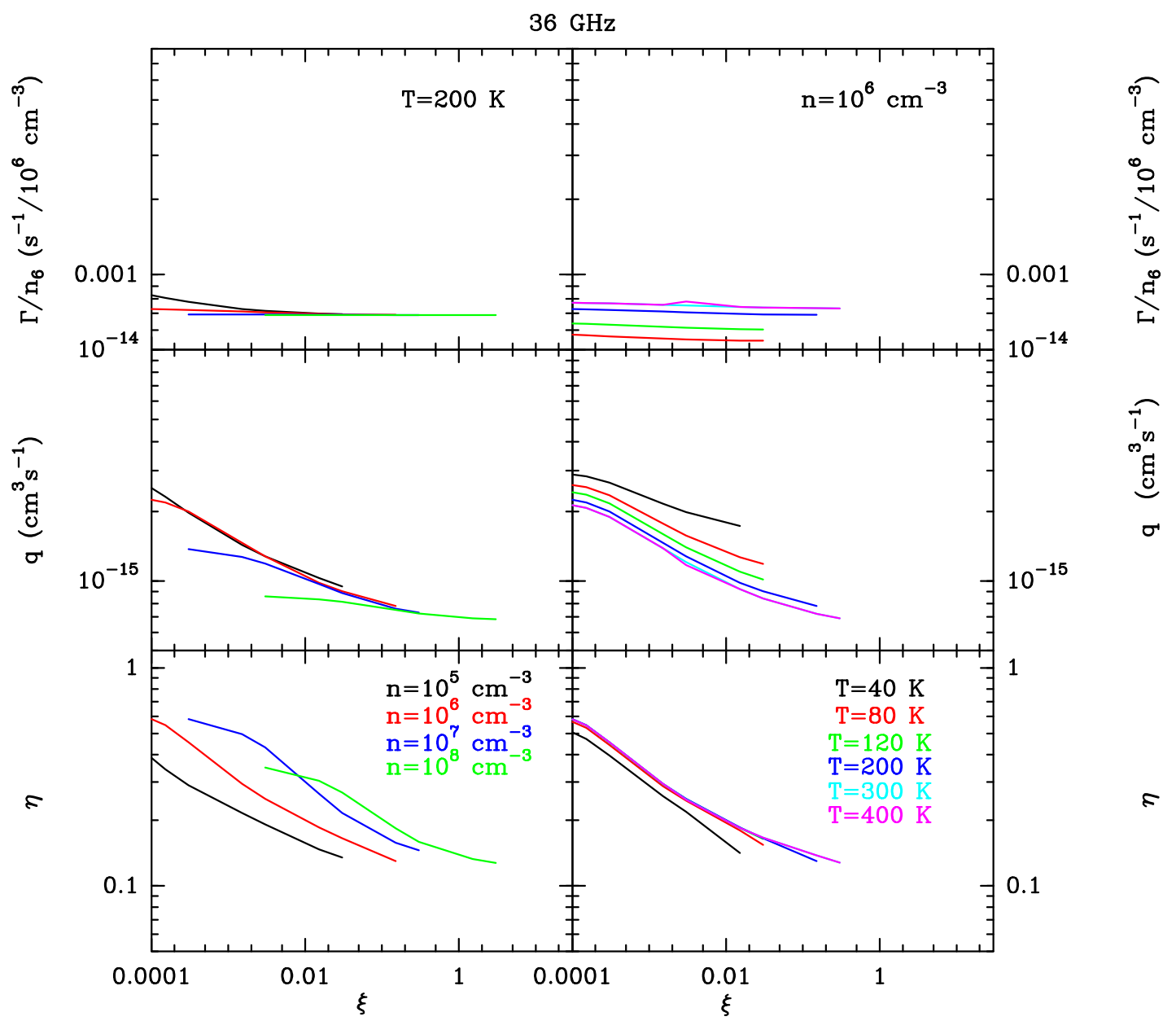

Fig. 8. Modelling results for the $36 \mathrm{GHz}$ maser pumping at $T=200 \mathrm{~K}$ and various densities (left panel) and at $n=10^{6} \mathrm{~cm}^{-3}$ and several temperatures (right panel). Plotted are the maser loss rate $\Gamma$ divided by $n_{6}$, the pump rate coefficient $q$, and the inversion efficiency $\eta$ as functions of $\xi$. It is possible to convert $\xi$ to methanol abundance using Eq. (3).

However, we conclude that the Zeeman result certainly favours high densities (upwards of $10^{6} \mathrm{~cm}^{-3}$ ) in the masing gas.

\subsection{Maser saturation}

The intensity of a maser reaches saturation at a value $J_{\mathrm{s}}$ given by

$J_{\mathrm{s}}=\frac{g_{l}}{g_{l}+g_{u}} \frac{\Gamma}{B_{\mathrm{m}}}$

where $B_{\mathrm{m}}$ is the Einstein coefficient for stimulated emission of the maser transition and $\Gamma$ the loss rate (Eq. (7)). The corresponding brightness temperature $T_{\mathrm{s}}$ of the line is

$T_{\mathrm{s}}=J_{\mathrm{s}} \times \frac{\lambda_{\mathrm{m}}^{2}}{2 k_{\mathrm{B}}}=\frac{\Gamma}{A_{\mathrm{m}}} \times \frac{h v_{\mathrm{m}}}{k_{\mathrm{B}}} \times \frac{g_{l}}{g_{l}+g_{u}}$,

where $\lambda_{\mathrm{m}}$ and $\nu_{\mathrm{m}}$ are the wavelength and frequency of the maser line, $k_{\mathrm{B}}$ the Boltzmann constant, $h$ the Planck constant, and $A_{\mathrm{m}}$ the Einstein coefficient for spontaneous emission of the maser transition. In this way, we do not correct for any given geometry to keep results general. Using $A_{44 \mathrm{GHz}}=2.0 \times 10^{-7} \mathrm{~s}^{-1}, A_{95 \mathrm{GHz}}=$ $2.1 \times 10^{-6} \mathrm{~s}^{-1}, A_{36 \mathrm{GHz}}=1.5 \times 10^{-7} \mathrm{~s}^{-1}, A_{84 \mathrm{GHz}}=2.0 \times 10^{-6} \mathrm{~s}^{-1}$, and $A_{25.018 \mathrm{GHz}}=8.6 \times 10^{-8} \mathrm{~s}^{-1}$ (from the Cologne database for molecular spectroscopy; Müller et al. 2001, 2005), we find brightness temperatures at saturation up to approximately $10^{5} \mathrm{~K}$ for the $44 \mathrm{GHz}, 95 \mathrm{GHz}, 36 \mathrm{GHz}$, and $84 \mathrm{GHz}$ lines for a temperature of $200 \mathrm{~K}$ and a density of $10^{8} \mathrm{~cm}^{-3}$. The $6_{2}-6_{1}-E$ has lower values of $T_{\mathrm{s}}(\sim 5000 \mathrm{~K})$. We show in Figs. 13 and 14 our computed values for $J_{\mathrm{s}}$ and $T_{\mathrm{s}}$ as a function of $\xi$ for a range of temperatures and densities. For the $44 \mathrm{GHz}, 95 \mathrm{GHz}, 36 \mathrm{GHz}$, and $84 \mathrm{GHz}$ masers, we find similar behaviour; $T_{\mathrm{s}}$ increases with the volume density of the gas. The $25 \mathrm{GHz}$ lines behave differently and show very little dependence on density. All lines are basically unaffected by changes in temperature. Moreover, the $T_{\mathrm{s}}$ of the masers scale with the density of the gas approximately as $4 \times 10^{3} n_{6}$ for the $44 \mathrm{GHz}$, and as $3 \times 10^{3} n_{6}$ for the $36 \mathrm{GHz}$ transition, and the $T_{\mathrm{s}}$ of the $95 \mathrm{GHz}$ and $84 \mathrm{GHz}$ lines can be obtained from the same relations corrected by a factor $(44 \mathrm{GHz} / 95 \mathrm{GHz})^{2}$ and $(36 \mathrm{GHz} / 84 \mathrm{GHz})^{2}$, respectively. From these relations, it is possible to derive constraints on the beaming angle $\Omega_{\mathrm{b}}$ of a given maser: if brightness temperatures of $10^{9} \mathrm{~K}$ are observed, as in IRAS 18151-1208 for the $44 \mathrm{GHz}$ line (Matsumoto et al. 2014), then assuming that the density of the gas is $\sim 10^{8} \mathrm{~cm}^{-3}, \Omega_{\mathrm{b}}$ is larger than $5 \times 10^{-3}$ for a saturated maser. Similar values of $\Omega_{b}$ are estimated for the $25.018 \mathrm{GHz}$ line $\left(\Omega_{\mathrm{b}}=0.03-0.003\right.$ for $T_{\mathrm{b}}=10^{6}-10^{7} \mathrm{~K}$; see Table 2).

\subsection{Comparison with observations}

In Sect. 2.3 we speculated that the Class I masers with the lowest excitation requirements are saturated, as suggested by their 


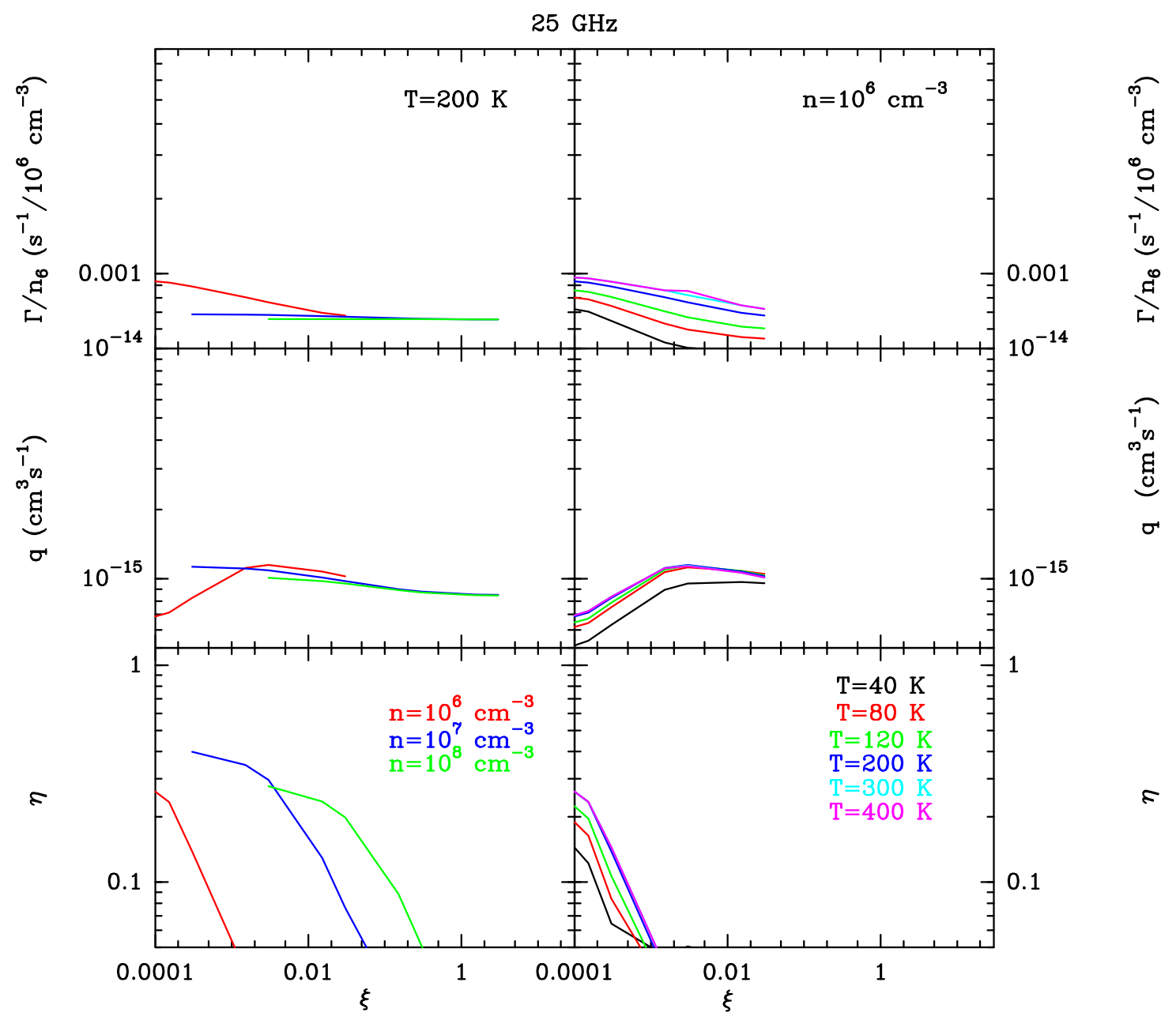

Fig. 9. Modelling results for the $6_{2}-6_{1}-E 25.018 \mathrm{GHz}$ maser pumping at $T=200 \mathrm{~K}$ and various densities (left panel) and at $n=10^{6} \mathrm{~cm}^{-3}$ and several temperatures (right panel). Plotted are the maser loss rate $\Gamma$ divided by $n_{6}$, the pump rate coefficient $q$, and the inversion efficiency $\eta$ as functions of $\xi$. It is possible to convert $\xi$ to methanol abundance using Eq. (3).

slow variability (in general) and by the similar velocity profiles of different transitions found in several sources. Under this assumption, and assuming that different lines are emitted by the same volume of gas, we can compare observed line ratios to our model results to verify whether our calculations reproduce the observations. Recently, Voronkov et al. (2014) have reported observations of a sample of 71 Class I maser methanol sources in the $36 \mathrm{GHz}$ and $44 \mathrm{GHz}$ lines with the ATCA array. These authors find that the $44 \mathrm{GHz}$ to $36 \mathrm{GHz}$ flux-density ratio has a wide distribution with a peak at 2.5 (corresponding to $\Phi_{44 \mathrm{GHz}} / \Phi_{95 \mathrm{GHz}} \sim 3$ ); only $14 \%$ of the 292 maser groups discussed by Voronkov et al. (2014) were found to be stronger at $36 \mathrm{GHz}$ than at $44 \mathrm{GHz}$ (see their Fig. 6). The authors also comment that owing to the complexity of spectral profiles, this is likely an upper limit to the number of groups where the $36 \mathrm{GHz}$ flux is stronger than at $44 \mathrm{GHz}$. These results seem to reflect our model predictions, which show that the $44 \mathrm{GHz}$ line is always stronger than the $36 \mathrm{GHz}$ maser except at low temperatures and high densities (see Figs. 10 and 12). These conditions are probably met in regions with little or no star formation activity. Indeed Pratap et al. (2008) reported enhanced maser emission at $36 \mathrm{GHz}$ relative to the $44 \mathrm{GHz}$ maser line toward such regions. Moreover, observations of supernovae remnants find $36 \mathrm{GHz}$ maser emission in the absence of a detectable $44 \mathrm{GHz}$ line (e.g., Pihlström et al. 2014). Regarding other flux ratios, no large sample of sources observed at high angular resolution is available. Fontani et al. (2010) detected the $44 \mathrm{GHz}$ and the $95 \mathrm{GHz}$ masers in a sample of high-mass star forming regions with the Nobeyama telescope. Seven sources in the sample are detected in both lines in a similar range of velocities and with similar line widths. Their observed $\Phi_{44 \mathrm{GHz}} / \Phi_{95 \mathrm{GHz}}$ ratio has an average value of $\sim 5$ and ranges between 1 and 11 , while the modelled ratio is never higher than 1. Clearly, our modelled $\Phi_{44 \mathrm{GHz}} / \Phi_{95 \mathrm{GHz}}$ fails to reproduce the observations. However, a note of caution is needed in the case of single-dish observations because the maser locations can be significantly offset from the pointing positions and because of the different beam sizes of the different lines $\left(38^{\prime \prime}\right.$ and $18^{\prime \prime}$ for the observations reported by Fontani et al. 2010).

Concerning the relative intensities of the $J_{2} \rightarrow J_{1}$ lines at $25 \mathrm{GHz}$, it is noteworthy that our calculations predict that the ratio between the photon production rates of the $6_{2} \rightarrow 6_{1}$ line to the $3_{2} \rightarrow 3_{1}$ transition is close to 3 and slightly increases with density (Fig. 11), in reasonable agreement with observations (Menten et al. 1986). These observations also show that the $J_{2} \rightarrow J_{1}$ lines usually peak at $J=6$, while our results show a peak around $J=9$. This may be the effect of the relatively high kinetic temperature we adopted for our calculations, $200 \mathrm{~K}$.

Another implication of our results is that in sources where the $25 \mathrm{GHz}$ lines are seen in absorption (e.g., W3OH, NGC 7538 Menten et al. 1986) the density must be lower than $10^{6} \mathrm{~cm}^{-3}$, and the $36 \mathrm{GHz}$ and $44 \mathrm{GHz}$ masers should be detected. This 


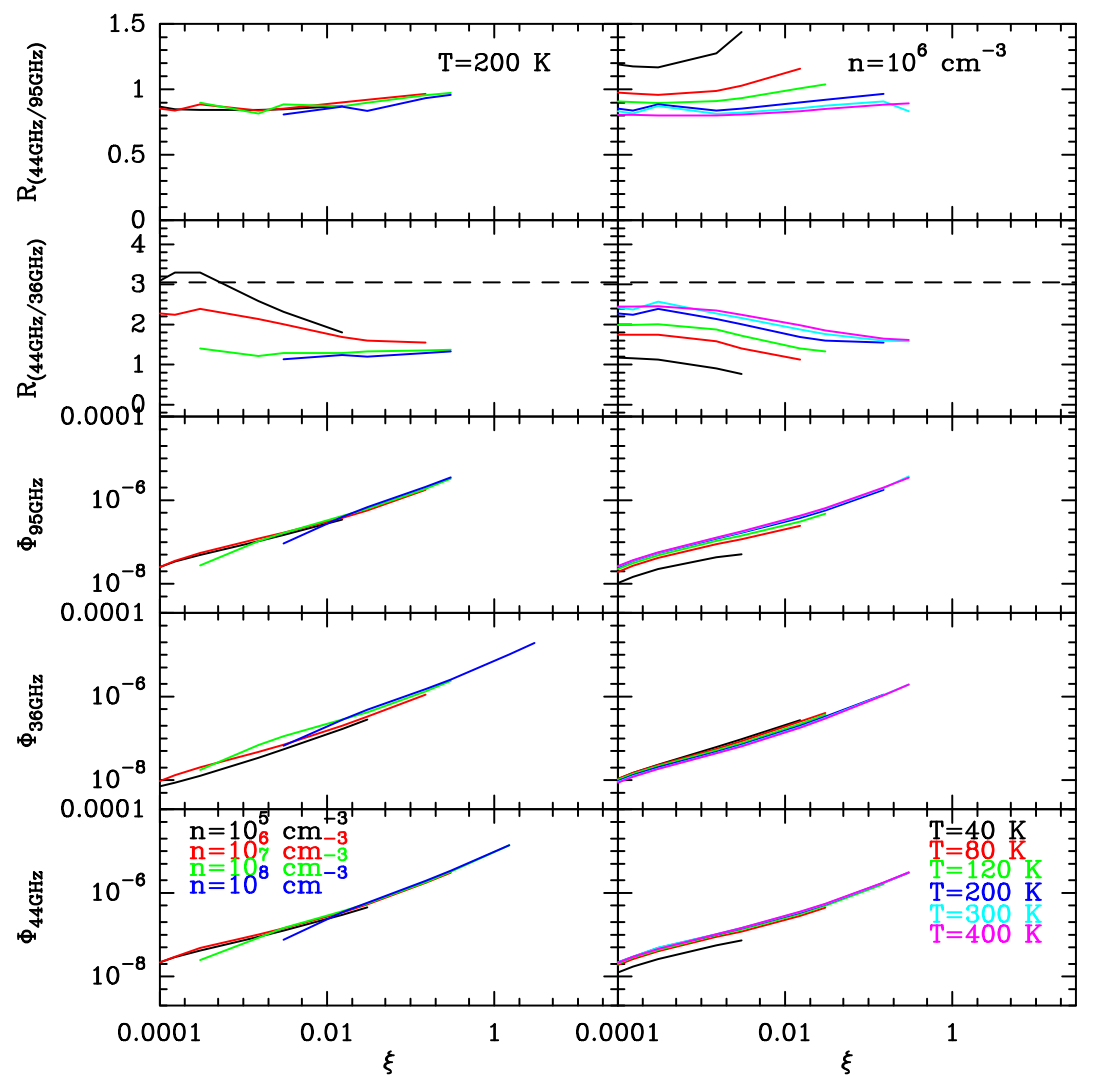

Fig. 10. Modelling results for the $44 \mathrm{GHz}, 36 \mathrm{GHz}$, and $95 \mathrm{GHz}$ maser photon production rates, and for their ratios at $200 \mathrm{~K}$ for various densities (left panel) and at $n=10^{6} \mathrm{~cm}^{-3}$ and several temperatures (right panel). The horizontal dashed line in the panels showing the $44 \mathrm{GHz}$ to $36 \mathrm{GHz}$ ratio marks the peak value of the flux-density ratio distribution reported by Voronkov et al. (2014). It is possible to convert $\xi$ to methanol abundance using Eq. (3). is indeed the case for both $\mathrm{W} 3 \mathrm{OH}$ and NGC 7538 (e.g., Haschick \& Baan 1989; Haschick et al. 1990; Kurtz et al. 2004), although the large beam of the single-dish observations does not allow verification of the spatial association of the lines.

Our results show that interferometric surveys in the $25 \mathrm{GHz}$ lines and in the millimetre masers (at $84 \mathrm{GHz}$ and $95 \mathrm{GHz}$ ) would be very useful. Figure 12 shows a comparison of our computed photon production rates at $200 \mathrm{~K}$ for densities between $10^{6}$ and $10^{8} \mathrm{~cm}^{-3}$. One interpretation of the small minority of objects with high $44 \mathrm{GHz}$ to $36 \mathrm{GHz}$ flux-density ratio might be that they are high emission measure (methanol abundance) objects with $\xi$ larger than 0.1 and density above $10^{7} \mathrm{~cm}^{-3}$. Certainly, a good interferometric survey is needed at $25 \mathrm{GHz}$ and in the $\mathrm{mm}$ lines to make further progress.

\section{Outlook}

Refining estimates of the beaming angle and related quantities will require a proper resolution of the radiative transport problem for a plausible geometry as well as a more consistent set of interferometric data. This will allow a more reliable estimate of the expected brightness temperature and line profile. To what extent does finite inclination of the shock propagation direction to the line of sight affect observed profiles? Is shock structure important and does this affect the observed maser structure? What angular size differences are expected between different Class I masers? Answers to these questions will become important for the new generation of interferometric results from the VLA and ALMA, for example.

As noted above, we conclude that bright Class I methanol masers are mainly high-temperature high-density structures with methanol maser emission measures $\xi$ close to the limits set by collisional quenching (roughly a hydrogen density of $\left.10^{8} \mathrm{~cm}^{-3}\right)$. The basic physics is very similar to that discussed by Hollenbach et al. (2013) for water masers and we find, for example, that in the case of methanol (as for water), the maser efficiency at high density is determined by the collisional selection rules rather than the relative radiative decay rates from the upper and lower maser levels. The fall-off of maser efficiency $\eta$ with $\xi$ is similar to that found by Hollenbach et al. (2013) for water masers, as is the increase of the saturated maser photon production rate $\Phi$ with $\xi$. Thus, relatively high post shock densities and temperatures (though not sufficiently high to cause dissociation of $\mathrm{H}_{2}$ ) are needed to account for most bright Class I masers. The results of Momjian \& Sarma (2012) discussed above suggest that magnetic fields may be important in the dynamics of the structures that give rise to methanol masers, and we conclude that $\mathrm{C}$-shocks (rather than $J$-shocks) give rise to Class I masers. The linear sizes observed (10-100 AU, see Table 2) are consistent with this. Kaufman \& Neufeld (1996) have studied the structure of $\mathrm{C}$-shocks in the parameter range of interest to us and find shock widths of roughly $10 \mathrm{AU}$ (dependent on preshock density, ionisation degree, etc.) with compression factors (post shock/pre-shock density ratio) of the order of the Alfven Mach number $v_{\mathrm{sh}} / v_{\mathrm{a}}$ (where $v_{\mathrm{sh}}$ is the shock velocity and $v_{\mathrm{a}}$ the Alfven velocity). The post-shock temperatures are of the order of a few hundred $\mathrm{K}$ and the Alfven Mach number is typically a factor of 10 . Thus for post-shock densities of the order of $10^{7} \mathrm{~cm}^{-3}$, we expect pre-shock densities of the order of $10^{6} \mathrm{~cm}^{-3}$. Hence, we can conclude that saturated Class I masers are likely to occur in regions of high ambient density and pressure. Highmass star forming regions are thus a more likely locale for Class I methanol masers than lower pressure clouds close to the Sun.

In this context, calculations based on a C-shock geometry (analogous to those of Hollenbach et al. 2013, and 
S. Leurini et al.: Bright Class I methanol masers

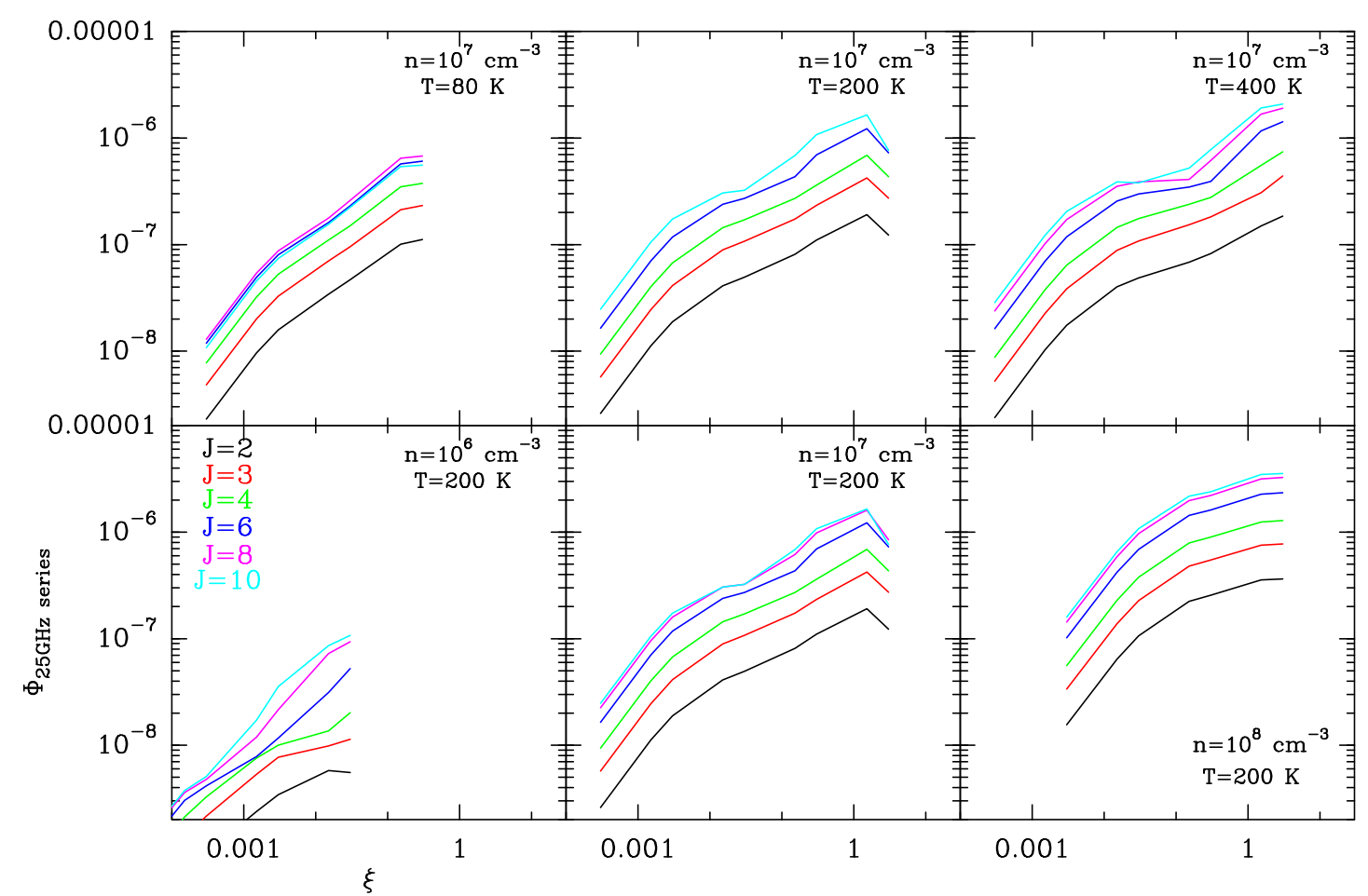

Fig. 11. Modelling results for the photon production rate of the $J_{2}-J_{1}-E(J=2-10)$ series at $25 \mathrm{GHz}$ maser at $200 \mathrm{~K}$ for various densities (bottom panel) and at $n=10^{7} \mathrm{~cm}^{-3}$ and several temperatures (upper panel). It is possible to convert $\xi$ to methanol abundance using Eq. (3).

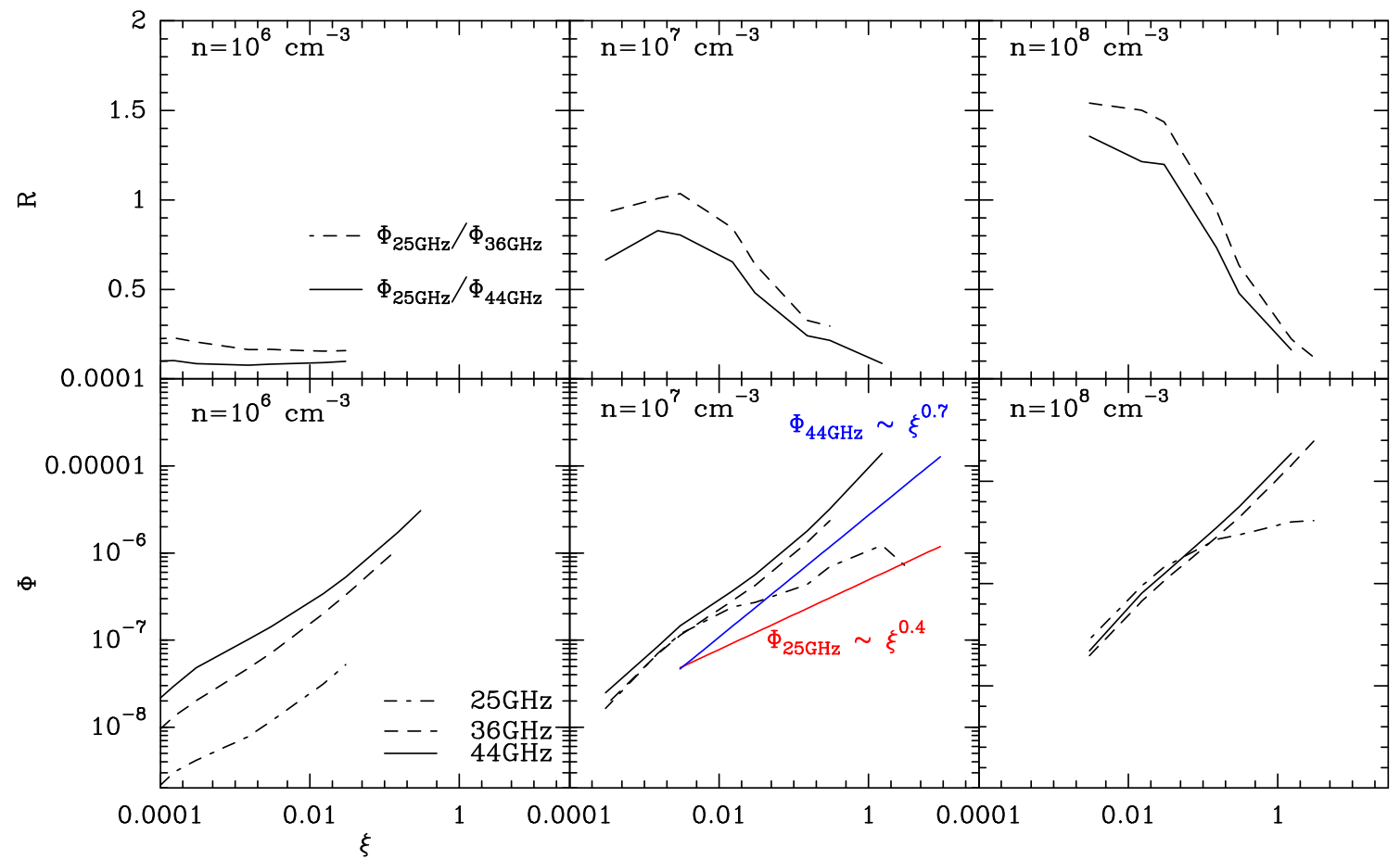

Fig. 12. Bottom panel: comparison of the maser photon production rates for the $44 \mathrm{GHz}, 36 \mathrm{GHz}$, and $25 \mathrm{GHz}(J=6)$ for various densities at $200 \mathrm{~K}$. For example, the solid red and blue curves in the middle panel show a power law of $\xi^{0.4}$ and $\xi^{0.7}$, respectively. Top panel: modelling results for the ratios of the $6_{2}-6_{1}-E$ maser photon production rate to that of the $44 \mathrm{GHz}$ and $36 \mathrm{GHz}$ lines for various densities at $200 \mathrm{~K}$. It is possible to convert $\xi$ to methanol abundance using Eq. (3).

Kaufman \& Neufeld 1996) are clearly needed to produce brightness temperature and line profiles for Class I masers that can be compared with observations. The computations of maser efficiency and photo production rate in this article are a first step. A comprehensive set of interferometric data which allow study of the spatial relationship between maser spots and their development in time is also clearly needed. In view of the importance of magnetic fields, calculations taking account of all Stokes parameters as well as more Zeeman measurements are needed as well. The essential first step here is to obtain reliable determinations of the Landé factors for the different maser transitions. 


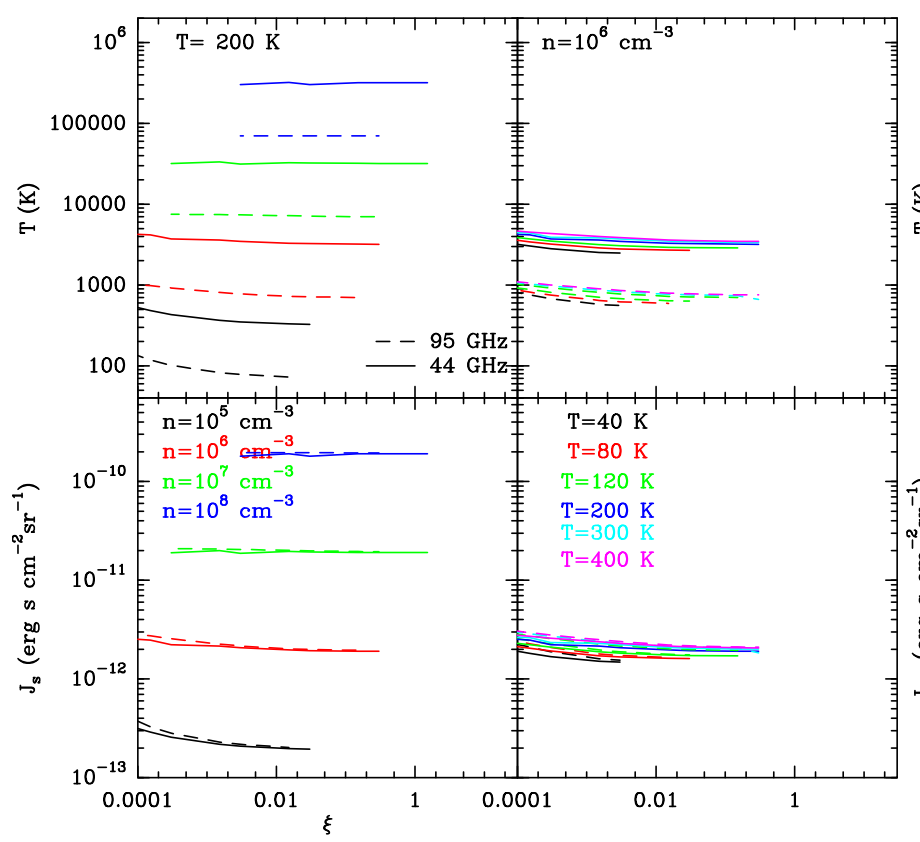

(a)

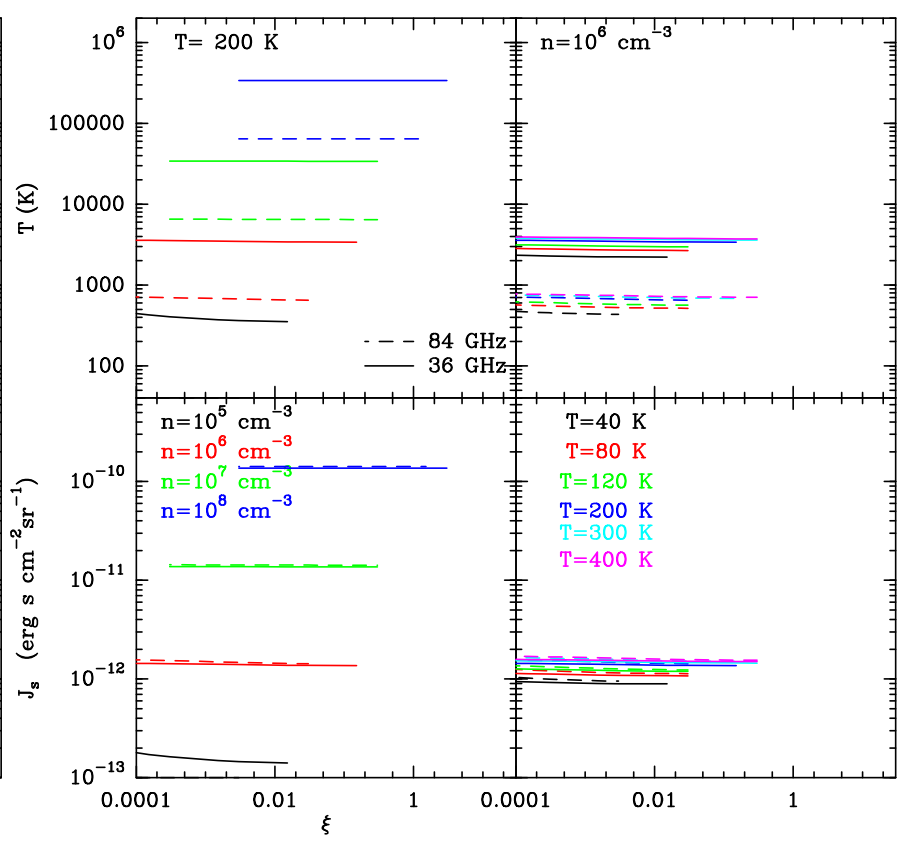

(b)

Fig. 13. $J_{\mathrm{s}}$ and $T_{\mathrm{s}}$ as function of $\xi$ for different densities and temperatures for the $44 \mathrm{GHz}$ (solid line) and $95 \mathrm{GHz}$ (dashed lines) transitions a), and for the $36 \mathrm{GHz}$ (solid line) and $84 \mathrm{GHz}$ (dashed lines) masers b). It is possible to convert $\xi$ to methanol abundance using Eq. (3).

Another important question to be investigated is the possible influence of $\mathrm{o}-\mathrm{H}_{2}$ collisions on the properties of Class I masers. It is unclear what the ortho-to-para abundance ratio will be in the shocks we postulate, but it is clear that $\mathrm{o}-\mathrm{H}_{2}$ collisions have different selection rules than $\mathrm{p}-\mathrm{H}_{2}$ collisions (Rabli \& Flower 2010) and that, if the $\mathrm{o} / \mathrm{p}$ ratio is approximately equal to or greater than 1 , this will change our results appreciably. It could conceivably switch off the maser. Thus, calculations assuming different $\mathrm{o} / \mathrm{p}$ ratios are needed.

One important consequence of such studies is obtaining direct observational information about $\mathrm{C}$-shock structure. Masers allow this to be studied both in time and space. It seems likely that it will be possible to measure proper motions in Class I masers and also effects due to inhomogeneities in the pre-shock medium. There are presumably gradients in temperature and density along the direction of shock propagation and differences in the spatial distribution of Class I masers may provide a guide to this. Understanding C-shock structure on the other hand is a valuable input to studies of the interaction of protostellar outflows with their surroundings. We conclude that further work along these lines is needed.

\section{Summary and conclusions}

Using new collisional rates for collisions of $\mathrm{CH}_{3} \mathrm{OH}$ with para$\mathrm{H}_{2}$, we computed the pump and decay rates as well as the pump efficiency for the most commonly observed Class I methanol maser transitions with the aim of investigating the physical conditions leading to bright Class I masers in regions of massive star formation. This is, to our knowledge, the first time that calculations of this type have been carried out for methanol masers. We hope that our results can be applied to an appropriate model of the geometry of saturated Class I methanol masers in order to compute a realistic brightness temperature distribution and beaming solid angle. Ultimately, one hopes that this may allow insight into the structure and velocity field of the shocks

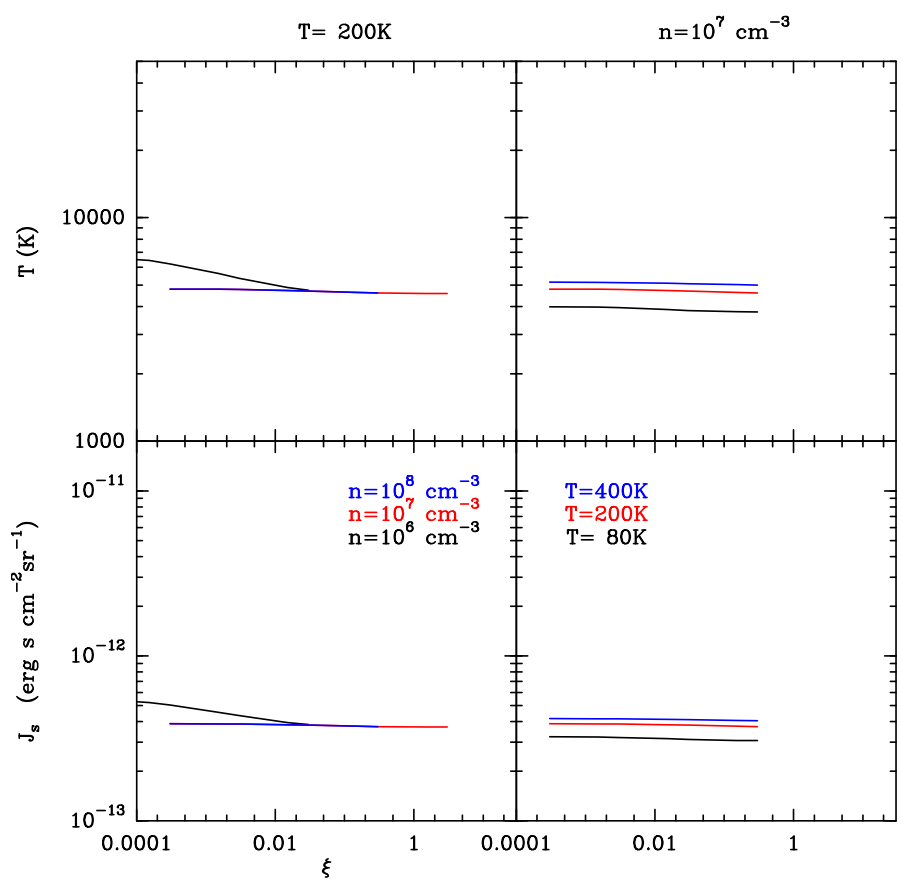

Fig. 14. $J_{\mathrm{s}}$ and $T_{\mathrm{s}}$ as function of $\xi$ for different densities and temperatures for the $6_{2}-6_{1}-E$ maser. It is possible to convert $\xi$ to methanol abundance using Eq. (3).

responsible for Class I maser emission. Our analysis of the photon production rates has allowed a few tentative conclusions on the properties of the high-gain masers such as those observed towards IRAS 18151-1208 by Matsumoto et al. (2014). They can be summarised as follows:

1. We predict inversion in all transitions where maser emission is observed. 
2. The production rates of all Class I masers increase with $\xi$ until thermalisation is reached. This implies that masers with high brightness temperature trace high methanol emission measure reached at high densities $\left(n\left(\mathrm{H}_{2}\right) \sim 10^{7}-10^{8} \mathrm{~cm}^{-3}\right)$, high temperatures $(>100 \mathrm{~K})$, and high methanol column densities with methanol maser emission measures close to the limits set by collisional quenching.

3. Class I masers can reasonably be separated into the three families: the $(J+1)_{-1}-J_{0}-E$ type series, the $(J+1)_{0}-J_{1}-A$ type, and the $J_{2}-J_{1}-E$ series at $25 \mathrm{GHz}$.

4. The $25 \mathrm{GHz}$ lines behave in a different way from the other maser transitions likely because they are inverted through $\Delta k \neq 0$ collisions. In particular, they are only inverted at high densities above $10^{6} \mathrm{~cm}^{-3}$ in contrast to other Class I masers, which mase already at densities of approximately $10^{4} \mathrm{~cm}^{-3}$.

5. Under the assumption that Class I masers are saturated, our calculations reproduce reasonably well most of the observed properties of Class I methanol masers in star forming regions. In particular, they predict that the $44 \mathrm{GHz}$ line is almost always stronger than the $36 \mathrm{GHz}$ maser, except at low temperatures and high densities and that the luminosity of the $25 \mathrm{GHz}$ series is lower than that of the other Class I masers. The observed constant ratio between different lines in the $25 \mathrm{GHz}$ series is also reproduced.

6. Maser emission in the $25 \mathrm{GHz}$ lines is not expected at low densities. Therefore, detection of all three families in the same maser feature is a clear indication of high densities $\left(>10^{6} \mathrm{~cm}^{-3}\right)$. On the other hand, detection of maser emission at $36 \mathrm{GHz}$ and $44 \mathrm{GHz}$ with no corresponding $25 \mathrm{GHz}$ masers indicates densities lower than $10^{6} \mathrm{~cm}^{-3}$ and probably larger size scales than the spot sizes of the order of $50 \mathrm{AU}$ observed in some sources.

7. The maser luminosities derived from our model predictions for the photon production rates are in agreement with observed isotropic luminosities for maser beam angles of the order of $10^{-3}$ steradian.

Acknowledgements. We thank the anonymous referee for helpful comments that have improved the content and overall clarity of this manuscript. C.M.W. would like to acknowledge travel support from the Science Foundation Ireland (Grant 13/ERC/I12907). C.M.W. acknowledges the hospitality of the MPIfR during the course of this work.

\section{References}

Araya, E. D., Kurtz, S., Hofner, P., \& Linz, H. 2009, ApJ, 698, 1321

Bachiller, R., Gomez-Gonzalez, J., Barcia, A., \& Menten, K. M. 1990, A\&A, 240, 116

Barrett, A. H., Schwartz, P. R., \& Waters, J. W. 1971, ApJ, 168, L101

Barrett, A. H., Ho, P., \& Martin, R. N. 1975, ApJ, 198, L119

Barrett, A. H., Hot, P. T. P., Martin, R. N., et al. 1976, Astrophys. Lett., 18, 13

Batrla, W., \& Menten, K. M. 1988, ApJ, 329, L117

Batrla, W., Matthews, H. E., Menten, K. M., \& Walmsley, C. M. 1987, Nature, 326,49

Breckenridge, S. M., \& Kukolich, S. G. 1995, ApJ, 438, 504

Britton, T. R., \& Voronkov, M. A. 2012, in IAU Symp. 287, eds. R. S. Booth, W. H. T. Vlemmings, \& E. M. L. Humphreys, 282

Buxton, R. B., Barrett, A. H., Ho, P. T. P., \& Schneps, M. H. 1977, AJ, 82, 985

Cesaroni, R., \& Walmsley, C. M. 1991, A\&A, 241, 537

Chen, X., Ellingsen, S. P., Shen, Z.-Q., Titmarsh, A., \& Gan, C.-G. 2011, ApJS, 196,9

Chui, M. F., Cheung, A. C., Matsakis, D., Townes, C. H., \& Cardiasmenos, A. G. 1974, ApJ, 187, L19

Cragg, D. M., Johns, K. P., Godfrey, P. D., \& Brown, R. D. 1992, MNRAS, 259, 203

Crutcher, R. M. 1999, ApJ, 520, 706

Cyganowski, C. J., Brogan, C. L., Hunter, T. R., \& Churchwell, E. 2009, ApJ, 702,1615

Davis, C. J., Kumar, M. S. N., Sandell, G., et al. 2007, MNRAS, 374, 29
Elitzur, M. 1992, Astronomical masers (Kluwer Academic Publishers) Elitzur, M., Hollenbach, D. J., \& McKee, C. F. 1989, ApJ, 346, 983 Ellingsen, S. P. 2005, MNRAS, 359, 1498

Ellingsen, S. P., Breen, S. L., Caswell, J. L., Quinn, L. J., \& Fuller, G. A. 2010 MNRAS, 404, 779

Ellingsen, S. P., Chen, X., Qiao, H.-H., et al. 2014, ApJ, 790, L28 Fish, V. L., Muehlbrad, T. C., Pratap, P., et al. 2011, ApJ, 729, 14 Fontani, F., Cesaroni, R., \& Furuya, R. S. 2010, A\&A, 517, A56 Frail, D. A. 2011, Mem. Soc. Astron. It., 82, 703

Gaines, L., Casleton, K. H., \& Kukolich, S. G. 1974, ApJ, 191, L99

Gan, C.-G., Chen, X., Shen, Z.-Q., Xu, Y., \& Ju, B.-G. 2013, ApJ, 763, 2 Goldreich, P., \& Kwan, J. 1974, ApJ, 189, 441

Green, J. A., Caswell, J. L., Fuller, G. A., et al. 2008, MNRAS, 385, 948 Green, J. A., Caswell, J. L., Fuller, G. A., et al. 2009, MNRAS, 392, 783 Gusdorf, A., Cabrit, S., Flower, D. R., \& Pineau Des Forêts, G. 2008, A\&A, 482, 809

Haschick, A. D., \& Baan, W. A. 1989, ApJ, 339, 949

Haschick, A. D., Menten, K. M., \& Baan, W. A. 1990, ApJ, 354, 556

Hills, R., Pankonin, V., \& Landecker, T. L. 1975, A\&A, 39, 149

Hollenbach, D., Elitzur, M., \& McKee, C. F. 2013, ApJ, 773, 70

Hunter, T. R., Brogan, C. L., Cyganowski, C. J., \& Young, K. H. 2014, ApJ, 788, 187

Johnston, K. J., Gaume, R., Stolovy, S., et al. 1992, ApJ, 385, 232

Johnston, K. J., Gaume, R. A., Wilson, T. L., Nguyen, H. A., \& Nedoluha, G. E. 1997, ApJ, 490, 758

Kalenskii, S. V., Bachiller, R., Berulis, I. I., et al. 1992, AZh, 69, 1002

Kalenskii, S. V., Liljestroem, T., Val'tts, I. E., et al. 1994, A\&AS, 103, 129

Kalenskii, S. V., Slysh, V. I., Val'Tts, I. E., Winnberg, A., \& Johansson, L. E. 2001, Astron. Rep., 45

Kalenskii, S. V., Johansson, L. E. B., Bergman, P., et al. 2010a, MNRAS, 405, 613

Kalenskii, S. V., Kurtz, S., Slysh, V. I., et al. 2010b, Astron. Rep., 54, 932

Kama, M., Dominik, C., Maret, S., et al. 2010, A\&A, 521, L39

Kaufman, M. J., \& Neufeld, D. A. 1996, ApJ, 456, 250

Kogan, L., \& Slysh, V. 1998, ApJ, 497, 800

Kurtz, S., Hofner, P., \& Álvarez, C. V. 2004, ApJS, 155, 149

Lees, R. M. 1973, ApJ, 184, 763

Lees, R. M., \& Haque, S. 1974, Can. J. Phys., 52, 2250

Lees, R. M., \& Oka, T. 1969, J. Chem. Phys., 51, 3027

Leurini, S., Schilke, P., Menten, K. M., et al. 2004, A\&A, 422, 573

Liechti, S., \& Wilson, T. L. 1996, A\&A, 314, 615

Lonsdale, C. J., Doeleman, S. S., Liechti, S., et al. 1998, BAAS, 30, 1355

Matsakis, D. N., Wright, M. C. H., Townes, C. H., et al. 1980, ApJ, 236, 481

Matsumoto, N., Hirota, T., Sugiyama, K., et al. 2014, ApJ, 789, L1

McEwen, B. C., Pihlström, Y. M., \& Sjouwerman, L. O. 2014, ApJ, 793, 133

Mehringer, D. M. \& Menten, K. M. 1997, ApJ, 474, 346

Mehrotra, S. C., Dreizler, H., \& Mäder, H. 1985, Naturforschung, 40, 683

Menten, K. 1991a, in Atoms, Ions and Molecules: New Results in Spectral Line Astrophysics, ASP Conf. Ser., 16, 119

Menten, K. M. 1991b, ApJ, 380, L75

Menten, K. M., \& Batrla, W. 1989, ApJ, 341, 839

Menten, K. M., Walmsley, C. M., Henkel, C., \& Wilson, T. L. 1986, A\&A, 157, 318

Menten, K. M., Johnston, K. J., Wadiak, E. J., Walmsley, C. M., \& Wilson, T. L. 1988a, ApJ, 331, L41

Menten, K. M., Reid, M. J., Moran, J. M., et al. 1988b, ApJ, 333, L83

Menten, K. M., Walmsley, C. M., Henkel, C., \& Wilson, T. L. 1988c, A\&A, 198, 267

Menten, K. M., Walmsley, C. M., Henkel, C., \& Wilson, T. L. 1988d, A\&A, 198 253

Menten, K. M., Reid, M. J., Pratap, P., Moran, J. M., \& Wilson, T. L. 1992, ApJ, 401, L39

Momjian, E., \& Sarma, A. P. 2012, AJ, 144, 189

Morimoto, M., Kanzawa, T., \& Ohishi, M. 1985, ApJ, 288, L11

Moscadelli, L., Menten, K. M., Walmsley, C. M., \& Reid, M. J. 1999, ApJ, 519, 244

Moscadelli, L., Goddi, C., Cesaroni, R., Beltrán, M. T., \& Furuya, R. S. 2007, A\&A, 472, 867

Müller, H. S. P., Thorwirth, S., Roth, D. A., \& Winnewisser, G. 2001, A\&A, 370, L49

Müller, H. S. P., Menten, K. M., \& Mäder, H. 2004, A\&A, 428, 1019

Müller, H. S. P., Schlöder, F., Stutzki, J., \& Winnewisser, G. 2005, J. Mol. Struct., 742,215

Nakano, M., \& Yoshida, S. 1986, PASJ, 38, 531

Nesterenok, A. V. 2016, MNRAS, 455, 3978

Pelling, M. 1975, MNRAS, 172, 41

Pihlström, Y. M., Sjouwerman, L. O., \& Fish, V. L. 2011, ApJ, 739, L21

Pihlström, Y. M., Sjouwerman, L. O., Frail, D. A., et al. 2014, AJ, 147, 73 
Plambeck, R. L., \& Menten, K. M. 1990, ApJ, 364, 555

Plambeck, R. L., \& Wright, M. C. H. 1988, ApJ, 330, L61

Pratap, P., Shute, P. A., Keane, T. C., Battersby, C., \& Sterling, S. 2008, AJ, 135, 1718

Rabli, D., \& Flower, D. R. 2010, MNRAS, 406, 95

Sarma, A. P., \& Momjian, E. 2009, ApJ, 705, L176

Sarma, A. P., \& Momjian, E. 2011, ApJ, 730, L5

Sanna, A., Moscadelli, L., Cesaroni, R., et al. 2010, A\&A, 517, A71

Sastry, K. V. L. N., Lees, R. M., \& de Lucia, F. C. 1984, J. Mol. Spectr., 103 486

Schilke, P., Walmsley, C. M., Pineau des Forets, G., \& Flower, D. R. 1997, A\&A, 321,293

Sjouwerman, L. O., Murray, C. E., Pihlström, Y. M., Fish, V. L., \& Araya, E. D. 2010a, ApJ, 724, L158

Sjouwerman, L. O., Pihlström, Y. M., \& Fish, V. L. 2010b, ApJ, 710, L111

Slysh, V. I., \& Kalenskii, S. V. 2009, Astron. Rep., 53, 519

Slysh, V. I., Kalenskij, S. V., \& Val'tts, I. E. 1993, ApJ, 413, L133

Slysh, V. I., Kalenskii, S. V., Valtts, I. E., \& Otrupcek, R. 1994, MNRAS, 268 464

Slysh, V. I., Kalenskii, S. V., Val'tts, I. E., \& Golubev, V. V. 1997, ApJ, 478, L37

Slysh, V. I., Kalenskii, S. V., Val'tts, I. E., Golubev, V. V., \& Mead, K. 1999, ApJS, 123, 515

Slysh, V. I., Kalenskii, S. V., \& Val'Tts, I. E. 2002, Astron. Rep., 46, 49

Sobolev, A. M. 1992, Sov. Astron., 36, 590

Sobolev, A. M., \& Deguchi, S. 1994a, ApJ, 433, 719

Sobolev, A. M., \& Deguchi, S. 1994b, A\&A, 291, 569

Sobolev, A. M., \& Strelnitskii, V. S. 1983, Sov. Astron. Lett., 9, 12

Sobolev, A. M., Cragg, D. M., \& Godfrey, P. D. 1997, A\&A, 324, 211

Sobolev, A. M., Wallin, B. K., \& Watson, W. D. 1998, ApJ, 498, 763

Szczepanski, J. C., Ho, P. T. P., Haschick, A. D., \& Baan, W. A. 1989, in The Center of the Galaxy, ed. M. Morris, IAU Symp., 136, 383
Tsunekawa, S., Ukai, T., Toyama, A., \& Takagi, K. 1995, Department of Physics, Toyama University, Japan, http://www.sci.u-toyama.ac.jp/ phys/4ken/atlas/

Urquhart, J. S., Moore, T. J. T., Menten, K. M., et al. 2015, MNRAS, 446, 3461 Val'tts, I. E., Dzyura, A. M., Kalenskii, S. V., et al. 1995, Astron. Rep., 39, 18 Val'tts, I. E., Ellingsen, S. P., Slysh, V. I., et al. 2000, MNRAS, 317, 315 van der Tak, F. F. S., Black, J. H., Schöier, F. L., Jansen, D. J., \& van Dishoeck, E. F. 2007, A\&A, 468, 627

Voronkov, M., Sobolev, A., Ellingsen, S., Ostrovskii, A., \& Alakoz, A. 2005, Ap\&SS, 295, 217

Voronkov, M. A., Brooks, K. J., Sobolev, A. M., et al. 2006, MNRAS, 373, 411 Voronkov, M. A., Brooks, K. J., Sobolev, A. M., et al. 2007, in IAU Symp. 242, eds. J. M. Chapman \& W. A. Baan, 182

Voronkov, M. A., Caswell, J. L., Britton, T. R., et al. 2010a, MNRAS, 408, 133 Voronkov, M. A., Caswell, J. L., Ellingsen, S. P., \& Sobolev, A. M. 2010b, MNRAS, 405, 2471

Voronkov, M. A., Walsh, A. J., Caswell, J. L., et al. 2011, MNRAS, 413, 2339

Voronkov, M. A., Caswell, J. L., Ellingsen, S. P., et al. 2012, in IAU Symp. 287, eds. R. S. Booth, W. H. T. Vlemmings, \& E. M. L. Humphreys, 433

Voronkov, M. A., Caswell, J. L., Ellingsen, S. P., Green, J. A., \& Breen, S. L. 2014, MNRAS, 439, 2584

Walmsley, C. M., Menten, K. M., Batrla, W., \& Matthews, H. E. 1988, A\&A, 197,271

Wilgenbus, D., Cabrit, S., Pineau des Forêts, G., \& Flower, D. R. 2000, A\&A, 356,1010

Wilson, T. L., Zeng, Q., Huettemeister, S., \& Dahmen, G. 1996, A\&A, 307, 209 Yanagida, T., Sakai, T., Hirota, T., et al. 2014, ApJ, 794, L10

Yusef-Zadeh, F., Cotton, W., Viti, S., Wardle, M., \& Royster, M. 2013, ApJ, 764, L19

Zapata, L. A., Loinard, L., Su, Y.-N., et al. 2012, ApJ, 744, 86

Zeng, Q., Lou, G. F., \& Li, S. Z. 1987, Ap\&SS, 132, 263 


\section{Appendix A: Additional figures}

Here we present the additional figures discussed in Sect. 4. We first present the excitation temperatures of the $36 \mathrm{GHz}, 84 \mathrm{GHz}$, and $95 \mathrm{GHz}$ masers as functions of $\xi$ for $200 \mathrm{~K}$ and for different densities to complement the plots for the other lines presented in the main text (Fig. A.1). Figures A.2-A.3 illustrate the maser loss rate $\Gamma$, the pump rate coefficient $q$, and the inversion efficiency $\eta$ as functions of $\xi$ for the $84 \mathrm{GHz}$ and $95 \mathrm{GHz}$ masers at various densities and temperatures. Finally, in Fig. A.4 we present the photon production rates of the $36 \mathrm{GHz}$ and $84 \mathrm{GHz}$ lines as functions of the methanol maser emission measures for different physical parameters.
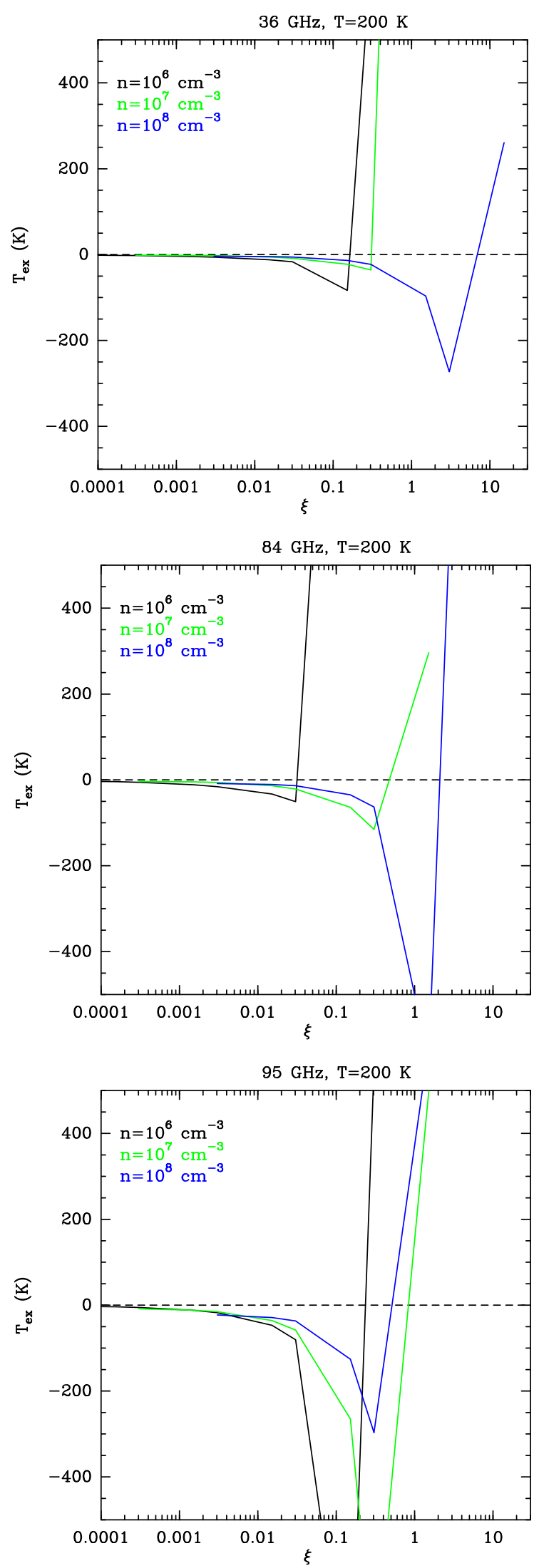

Fig. A.1. Excitation temperature of the $36 \mathrm{GHz}$ (upper panel), $84 \mathrm{GHz}$ (middle panel), and $95 \mathrm{GHz}$ lines (lower panel) at different densities and for a temperature of $200 \mathrm{~K}$. Curves are plotted until $T_{\mathrm{ex}}$ becomes positive. It is possible to convert $\xi$ to methanol abundance using Eq. (3). 
A\&A 592, A31 (2016)

$84 \mathrm{GHz}$

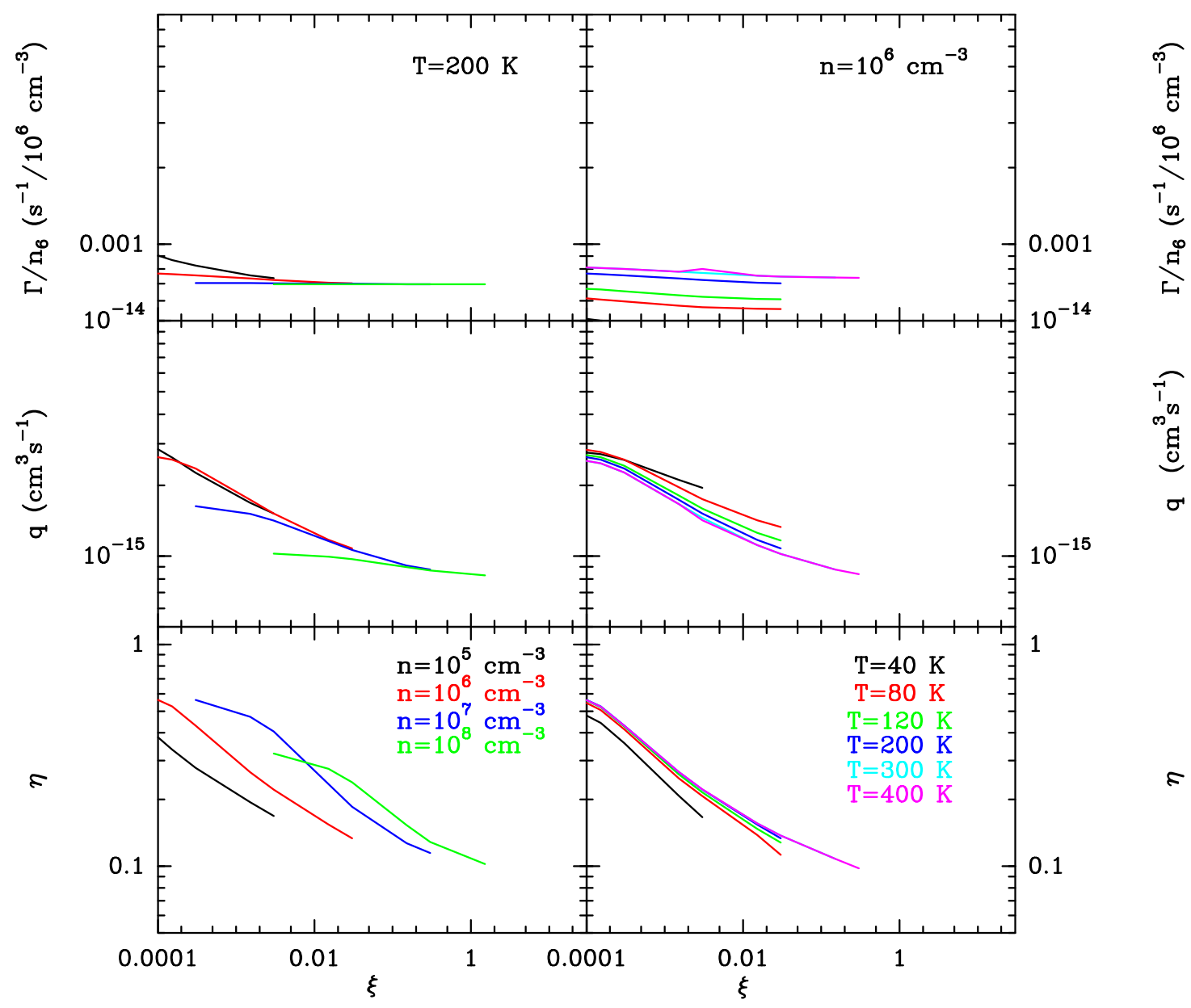

Fig. A.2. Modelling results for the $84 \mathrm{GHz}$ maser pumping at $T=200 \mathrm{~K}$ and various densities (left panel) and at $n=10^{6} \mathrm{~cm}^{-3}$ and several temperatures (right panel). Plotted are the maser loss rate $\Gamma$ divided by $n_{6}$, the pump rate coefficient $q$, and the inversion efficiency $\eta$ as functions of $\xi$. It is possible to convert $\xi$ to methanol abundance using Eq. (3). 
S. Leurini et al.: Bright Class I methanol masers

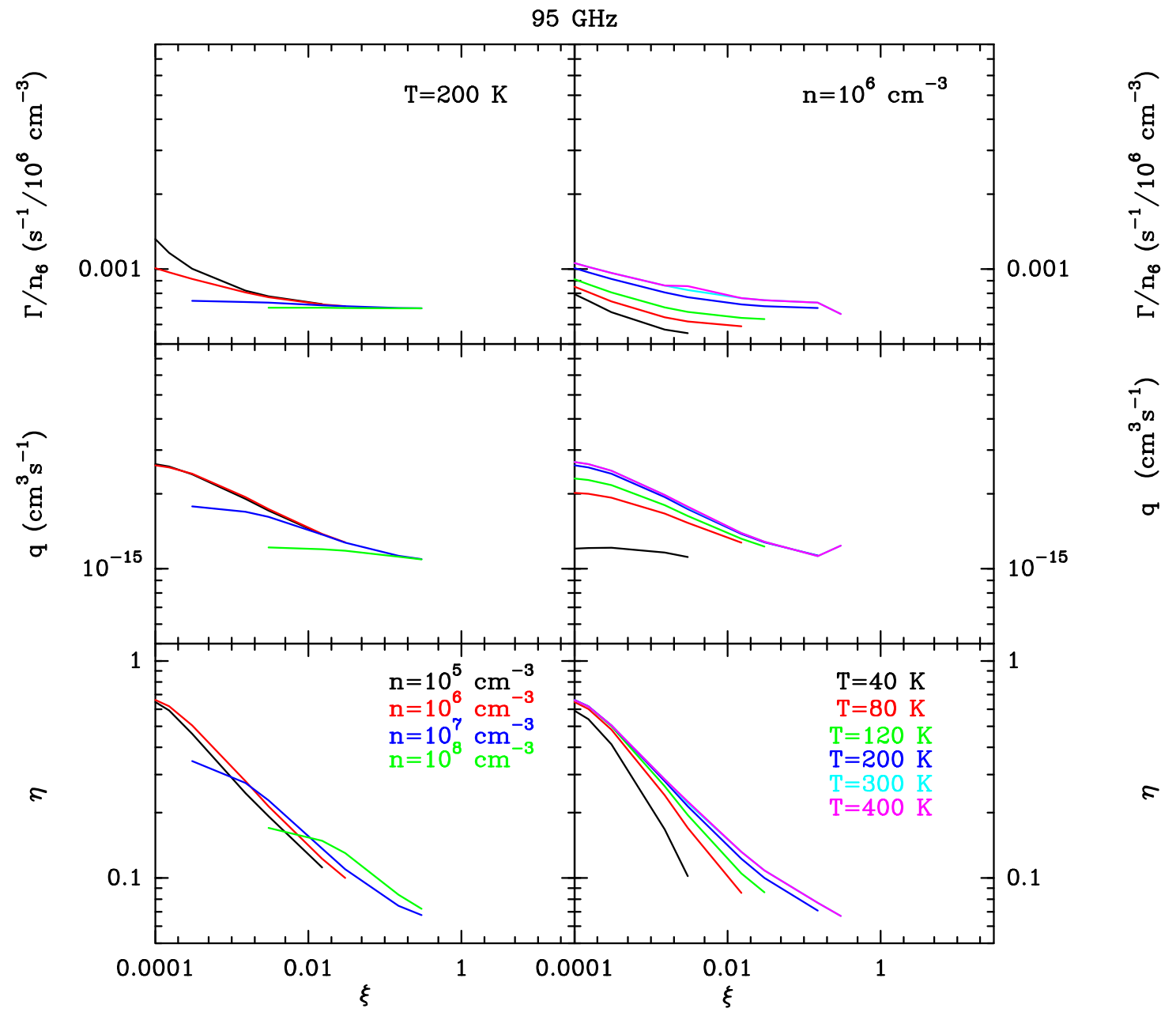

Fig. A.3. Modelling results for the $95 \mathrm{GHz}$ maser pumping at $T=200 \mathrm{~K}$ and various densities (left panel) and at $n=10^{6} \mathrm{~cm}^{-3}$ and several temperatures (right panel). Plotted are the maser loss rate $\Gamma$ divided by $n_{6}$, the pump rate coefficient $q$, and the inversion efficiency $\eta$ as functions of $\xi$. It is possible to convert $\xi$ to methanol abundance using Eq. (3). 
A\&A 592, A31 (2016)

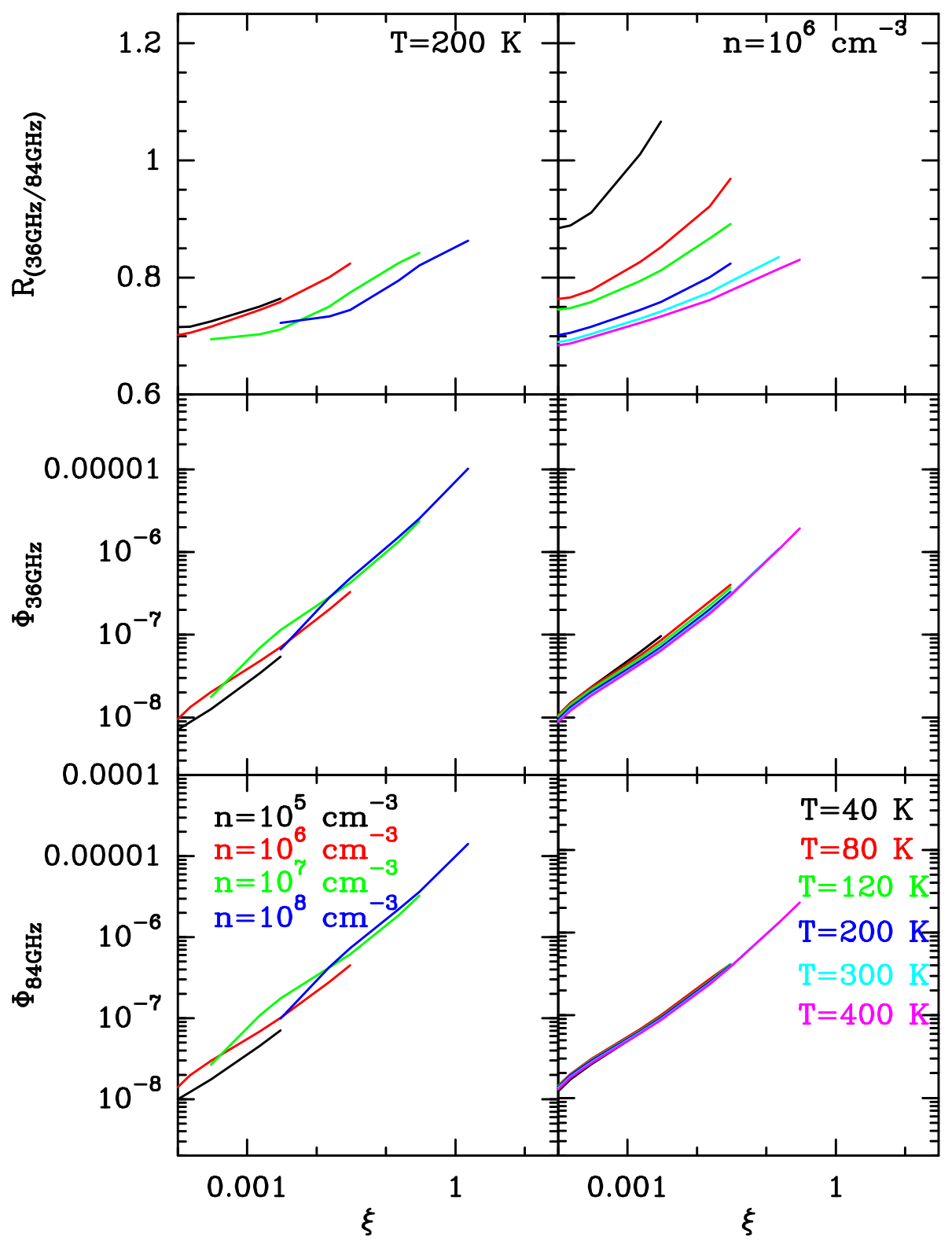

Fig. A.4. Modelling results for the $36 \mathrm{GHz}$ (bottom) and $84 \mathrm{GHz}$ (middle) maser photon production rates, and for their ratios (top) at $200 \mathrm{~K}$ for various densities (left panel) and at $n=10^{6} \mathrm{~cm}^{-3}$ and several temperatures (right panel). It is possible to convert $\xi$ to methanol abundance using Eq. (3). 